\title{
A Developmentally Regulated Antigen Associated with Neural Cell and Process Migration
}

\author{
Rosalia Mendez-Otero, ${ }^{a}$ Burkhard Schlosshauer, ${ }^{b}$ Colin J. Barnstable, ${ }^{1}$ and Martha Constantine-Paton \\ Department of Biology, Yale University, New Haven, Connecticut 06511, and 'Laboratory of Neurobiology, The \\ Rockefeller University, New York, New York 10021
}

The distribution of an epitope recognized by the monoclonal antibody JONES has been studied immunohistochemically in the developing nervous system of the rat. In the present report, we survey selected regions of the fetal, postnatal, and adult rat nervous system to test the hypothesis that JONES binding is invariably associated with neural cell migration and axon growth in the developing rat.

A series of selected developmental stages extending from embryonic day (E) 9 to adult were used in this investigation. The distribution of JONES binding was examined using indirect immunofluorescence, as well as the immunogold procedure. Particular attention was paid to regions where the positions and timing of cell and axon migrations have been well described for the rat. JONES immunoreactivity first appears at E11-12, when it is localized to the lamina terminalis, the telencephalic-diencephalic junction, the midbrain, and the rhombic lip regions of the cytologically undifferentiated neural tube. In all the regions studied, during embryonic and early postnatal life, the labeling is very Intense in the ventricular zone and shows a radial array in the adjacent intermediate and marginal zones. The expression of JONES epitope correlates particularly with times of cell migration in the retina, superior colliculus, cerebellum, and telencephaIon and in regions undergoing neurite extension, such as the developing optic tract, the white matter of the cerebellum, the dorsal roots, the trigeminal system, and olfactory nerve. JONES binding becomes progressively restricted in the postnatal period. In the adult brain, immunoreactivity is present only in the retina and cerebellum. In the retina, JONES labeling is present in the outer plexiform layer and optic fiber layer. The labeling in the optic fiber layer extends to the optic nerve head and stops abruptly outside the orbit. In the cerebellum, JONES shows a radially oriented pattern throughout the molecular layer and delineates the cell bodies

\footnotetext{
Received Mar. 13, 1987; revised Aug. 10, 1987; accepted Aug. 14, 1987.

We wish to thank Daniela Bonafede and Elizabeth Debski for their helpful comments on the manuscript and Leena Kalghati for excellent technical assistance. This work was supported by a Biomedical Rescarch Support Grant R07015 to Yale University, a NIH Grant NS 20483 to C. J. B., a Fulbright/Capes Fellowship Award and a Brazilian CNPq Fellowship to R.M.-O., and a Deutsche Forschungsgemeinschaft Fellowship to B.S.

Correspondence should be addreessed to Dr. Rosalia Mendez-Otero, Department of Biology, Yale University, Kline Biology Tower, P.O. Box 6666, New Haven, CT 06511.

* Permanent address: Universidade do Estado do Rio de Janeiro, Instituto de Biologia, Rio de Janeiro, RJ 20551 Brazil.

- Present address: Max-Planck-Institut für Entwicklungsbiologie, Spemannstr. 35/I, D-7400 Tübingen, Federal Republic of Germany.

Copyright (C) 1988 Society for Neuroscience $0270-6474 / 88 / 020564-16 \$ 02.00 / 0$
}

in the Purkinje cell layer. The only non-neural regions that show JONES immunoreactivity are the adrenal medulla and the kidney glomeruli.

We conclude that the antigens recognized by the JONES monoclonal antibody are associated with the migration of subsets of cells and axons within the developing rat nervous system and, consequently, may play a role in conveying selectivity to these processes.

Early events of brain differentiation are likely to be mediated, at least in part, by molecules that alter the surface properties of developing cell membranes in highly specific and stereotyped patterns (Gottlieb and Glaser, 1980). Thus, attempts to understand the mechanisms of cell interaction and recognition during neural development have been greatly facilitated by the production of highly specific antibody probes capable of localizing, identifying, and ultimately disrupting the normal functioning of the underlying molecules (see Valentino et al., 1985, for review).

Recently, we described (Constantine-Paton et al., 1986) a monoclonal antibody (JONES) that exhibits a highly regulated spatial and temporal pattern of immunoreactivity in developing rat retina. Immunoreactivity coincided with the appearance of the first postmitotic retinal cells and with the growth of retinal axons. In addition, the antibody showed a dorsal-ventral gradient of binding in the retina throughout the period when the topographic retinal projections are being established. These observations suggested that the antigen recognized by JONES might be important in conveying selectivity during the processes of neural cell migration and axon growth.

Biochemical characterization of the molecules carrying the JONES epitope reveals them to be the same 2 or 3 gangliosides in all regions examined (Schlosshauer et al., 1986, 1988). Members of this class of cell-surface glycolipids have been implicated in a wide variety of cell interactions (e.g., Marchase, 1977; Hakomori, 1981; Blackburn et al., 1986). Consequently, in order to examine the specific hypothesis that the JONES gangliosides are associated with cell and axon migration, we have mapped the changing distribution of JONES immunoreactivity in selected regions of the developing rat nervous system and asked how consistently it is associated with the patterns of neural cell migration and axon outgrowth that have been well described for the rat by previous investigators. A preliminary report of this work has already appeared (Mendez-Otero et al., 1986).

\section{Materials and Methods}

Antibody production. The production of the JONES monoclonal antibody (mab) was detailed previously (Constantine-Paton et al., 1986). Briefly, Balb/C mice received 3 intraperitoneal immunizations with 
fixed retinas from embryonic day (E) 13-20 rats. A final intravenous boost was given $4 \mathrm{~d}$ prior to the fusion of spleen cells with P3-NSI/1$\mathrm{Ag} 4-1$ plasmacytoma cells. The resulting hybridoma cell lines were screened immunohistochemically on sections of the retinas from E1314 and postnatal day (P) 1 rats using indirect immunofluorescence. The JONES antibody was selected for further analysis on the basis of its immunofluorescent staining pattern on sections of fixed embryonic and early postnatal rat brain and retina. The JONES mab was subcloned 3 times by limiting dilution, and antibody subclass (IgM) was determined by the Ouchterlony method (Ouchterlony and Nilsson, 1978).

Several series of early fetal rat brains were examined using concentrated hybridoma supernatant and immunofluorescence. However, all of the antibody used in the immunogold cytochemistry reported here and in the following biochemical description was the purified IgM fraction of ascites tumor fluid. This was produced by priming $\mathrm{Balb} / \mathrm{C}$ mice with Pristane $(300 \mu l) 4-14 \mathrm{~d}$ before the injection of $1 \times 10^{7}$ hybridoma cells intraperitoneally. The ascites fluid was collected 7-28 d after the hybridoma injection by washing the cavity with PBS. This fluid was centrifuged at $100,000 \times g$ at $4^{\circ} \mathrm{C}$ for $1 \mathrm{~h}$, and the supernatant was concentrated by ultrafiltration. Protein fractions were separated by gel filtration using a Sephacryl S-400 column. The purest IgM fraction was subsequently identified by SDS gel electrophoresis (Laemmli, 1970) and by immunohistochemical dilution series.

Animals. Initial studies with the JONES antibody indicated that it worked equally well on all rat strains. The earliest in vestigations used fetuses from pregnant female Long-Evans rats obtained from Charles River. However, for most of the work presented here Long-Evans or Sprague-Dawley rat females were mated with Black males in our breeding colony. The pregnancies were timed by testing vaginal smears for the presence of sperm. The first day on which sperm was detected was taken as day zero of gestation (E0) and the females were removed from the cages of the males. Postnatal day 0 refers to the first $24 \mathrm{hr}$ after birth. A series of developmental stages extending from E9 to adult were used in this study.

To obtain prenatal material, pregnant females were deeply anesthetized with ether or with an overdose of Nembutal and killed by cervical dislocation, and the uteri were dissected into ice-cold phosphate buffer (pH 7.2). E9 to E12 embryos were fixed by immersion into $4 \%$ paraformaldehyde in $0.1 \mathrm{~m}$ phosphate buffer for 4-6 $\mathrm{hr}$ at $4^{\circ} \mathrm{C}$. Embryos older than E12 and postnatal animals were perfused intracardially (under ether anesthesia) with $4 \%$ paraformaldehyde and postfixed in the same fixative for $4-8 \mathrm{hr}$ at $4^{\circ} \mathrm{C}$. The brains and eyes of postnatal animals were dissected out and transferred to phosphate buffer containing $30 \%$ sucrose for 12-48 hr. They were then embedded in OCT compund (Lab Tek), sectioned at $15 \mu \mathrm{m}$ on a Bright (Hacker Inst.) cryostat at $-20^{\circ} \mathrm{C}$ in the horizontal, coronal, or sagittal plane, mounted on chrome alum/ gelatin-subbed slides, and air-dried for $30 \mathrm{~min}$.

Immunohistochemistry. The distribution of the antigen in fixed frozen sections was demonstrated using indirect immunofluorescence or silverintensified immunogold staining procedures. In both cases, sections were incubated with the monoclonal antibody as full-strength supernatant or with purified IgM JONES antibody $(4 \mu \mathrm{g} / \mathrm{ml}$ in $5 \%$ goat serum) for $16-18 \mathrm{hr}$ at $4^{\circ} \mathrm{C}$. The slides were then rinsed 3 times with PBS or with $0.1 \% \mathrm{BSA}$-Tris solution $(0.1 \% \mathrm{wt} / \mathrm{vol} \mathrm{BSA}$ in Tris pH 8.2 and 20 mм $\mathrm{NaN}_{3}$ ). For indirect immunofluorescence sections were incubated with rhodamine isothiocyanate-conjugated goat IgG directed against mouse IgM (Cappel, 1:50 dilution). For the immunogold procedure, sections were incubated with the immunogold staining reagent (affinitypurified goat IgG directed against mouse IgM linked to $5 \mathrm{~nm}$ colloidal gold particles, Janssen, 1:40 dilution). Following exposure to the second antibody for $2 \mathrm{hr}$ at room temperature, sections were again rinsed in PBS. Rhodamine-labeled sections were mounted in glycerol, coverslipped, and examined under a Leitz epifluorescent photomicroscope. Sections incubated with the immunogold reagent were reacted with the silver-intensification kit (Janssen) to improve the visualization of the colloidal gold particles. Some of these sections were counterstained with toluidine blue. All immunogold-labeled sections were dehydrated, mounted with coverslips, and analyzed under dark- and bright-field microscopy. Control sections were incubated without the primary or secondary antibody. For each region, sections of animals of different ages were reacted at the same time to ensure that the differences in staining were not due to variations in the reaction. We have analyzed the brains of 2 or more animals at each stage.

A few sections from PO forebrain and P6 cerebellum were incubated with anti-D.1.1 antibody (a generous gift from Dr. W. B. Stallcup and
Dr. J. Levine) at 1:100 to 1:500 dilutions and reacted subsequently with rhodamine-conjugated secondary antibody.

\section{Results}

\section{Initial expression of JONES antigens}

Examination of early embryonic stages utilized indirect immunofluorescence as well as the immunogold procedure. These experiments failed to reveal JONES binding in primitive streak embryos and embryos determined to be at stage E9 and E11 by sperm-positive tests. The earliest detectable JONES binding was found in one series of brains for which exact gestational age was unknown. These embryos were morphologically similar to E11, suggesting that JONES immunoreactivity is first detectable on late E11 or early E12. At this stage, major brain regions were identifiable, but the walls of the neural tube still had the appearance of an undifferentiated pseudostratified epithelium. In both immunofluorescence and immunogold staining, JONES binding appeared around the perimeter of cells, occassionally with discrete puncta showing intense fluorescence (Fig. 1B). Intense staining at this stage was restricted to 3 regions: (1) the lamina terminalis and the ventral telencephalic and diencephalic regions immediately rostral and caudal to it; (2) the dorsal mesencephalon immediately caudal to the mesencephalic/diencephalic junction; and (3) the rhombic lip. These regions are illustrated in the camera lucidae drawings of sections shown in Figure $1 A$.

By E13, JONES binding was distributed widely in the forebrain, midbrain, and hindbrain. Immunoreactivity was intense in the ventricular zone, where cell bodies were present in high density. However, the sensitive immunogold staining procedure revealed that the intense ventricular zone binding was usually accompanied by less intense binding in the adjacent intermediate and marginal zones (see Fig. 8, $B, C$, for examples).

\section{Visual pathway}

Retina. The embryonic distribution of JONES immunoreactivity in retina has already been described (Constantine-Paton et al., 1986). Over the first $2-3$ postnatal days (P2-3), the retina is composed of a relatively uniform neuroblastic layer separated from the ganglion cell layer by a thin inner plexiform layer (Fig. $2 B$ ). JONES labeling can be detected throughout the whole retina, although the density of labeling in the 2 inner layers (ganglion cell and inner plexiform layers) is generally lighter than that in the outer layers (Fig. $2 A$ ). In this and all other stages studied, the optic fiber layer shows JONES immunoreactivity. However, no binding of this antibody was detected in the postnatal animal at the optic nerve head or in the optic nerve, 2 regions where ganglion cell axons are highly concentrated.

During the subsequent days, there is a progressive restriction in the radial distribution of JONES binding. This radial restriction parallels the laminar maturation pattern across the surface of the epithelium, being more advanced in the central than in the peripheral retina. By P7, the outer plexiform layer appears, and the inner region of the inner nuclear layer is well differentiated (Fig. 2D). The pattern of JONES binding at this age is shown in Figure $2 C$ : labeling is restricted to the outer two-thirds of the retina and to the optic fiber layer. Antibody binding has retracted from the inner and outer plexiform layers, as well as the inner half of the inner nuclear layer.

A marked drop in the intensity of the immunocytochemical reaction in retina occurs in the subsequent week. By P14, bind- 

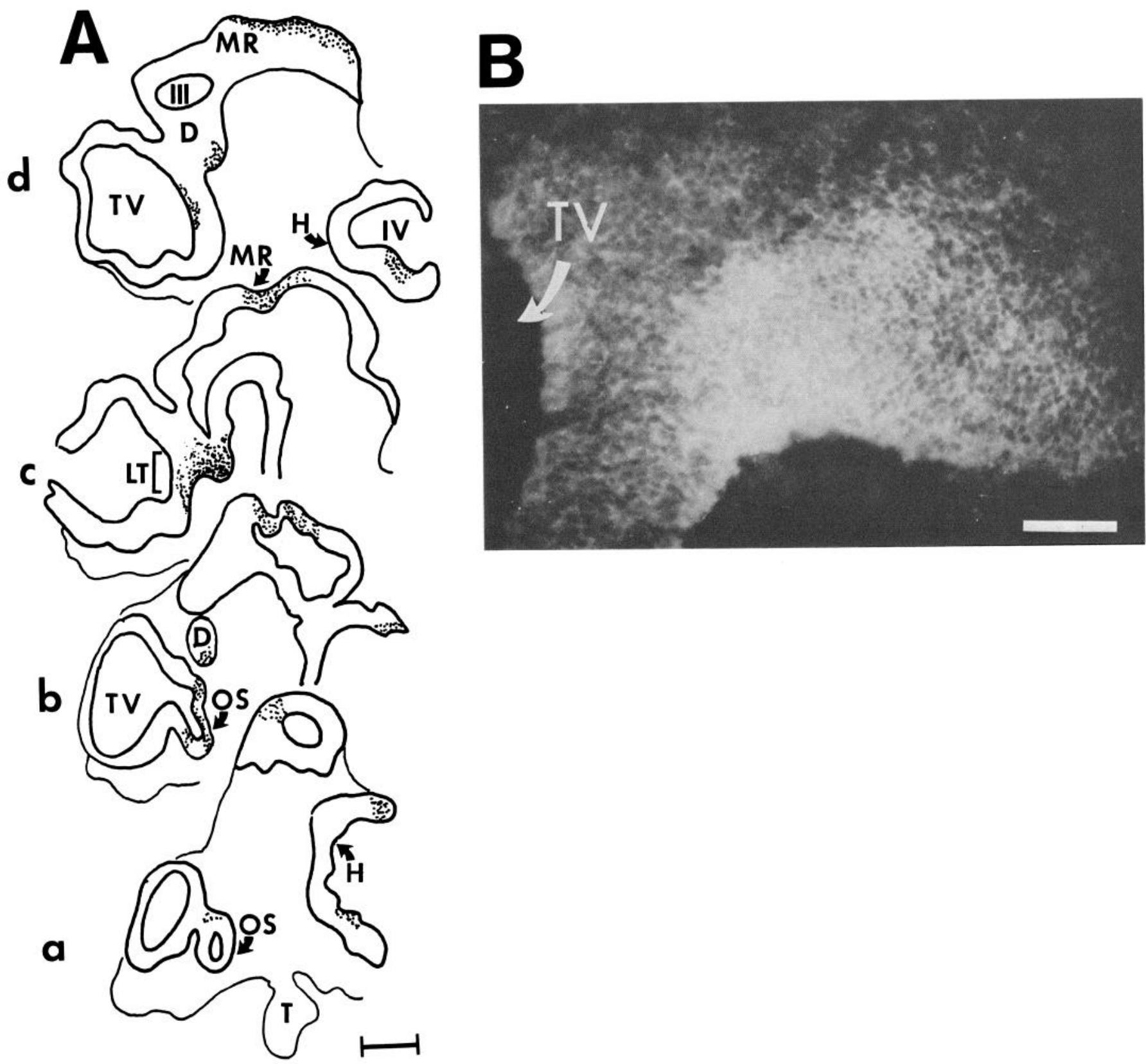

Figure 1. Pattern of JONES binding in the E11-12 brain. A, Series of camera lucida tracings of the early brain in sections cut close to the sagittal plane (e.g., sagittal but slightly tilted toward the horizontal plane). Section $a$ is the most lateral and $d$ the most medial. JONES immunoreactivity was visualized with indirect immunofluorescence. The position of JONES binding in different brain regions is indicated by stipple. The sections illustrated were chosen from a larger set of $42(15-17 \mu \mathrm{m})$ sections through the entire fetal head to illustrate the 3 regions where immunoreactivity first appears. Binding can be observed in the ventral forebrain at the telencephalic-diencephalic junction, in the dorsal midbrain beginning at the diencephalic-mesencephalic junction, and in the hindbrain region of the rhombic lip. Abbreviations: telencephalic vesicle (TV), optic stalk $(O S)$, lamina terminalis $(L T)$, diencephalon $(D)$, third ventricle $(I I I)$, mesencephalic roof $(M R)$, hindbrain $(H)$, fourth ventricle $(I V)$, and tongue $(T)$. $B$, High-power fluorescent photomicrograph of the same section illustrated in $d$ to show the pattern of labeling in the telencephalic-diencephalic region. In the less intensely stained regions, JONES binding can be distinguished around the perimeter of the cells and occasionally as brighter fluorescent puncta. The zone of JONES binding can be distinguished as a large, but contiguous, group of cells that show no morphological or cytological differences from cells in adjacent unlabeled regions. In both panels, rostral is to the left. Scale bar: $A, 300 \mu \mathrm{m} ; B, 50 \mu \mathrm{m}$.

ing is hardly visible in the central retina (Fig. $2 E$ ); however, it can still be detected in the peripheral retina, where it is localized to the outer retinal regions and the optic fiber layer (Fig. $2 F$ ).

Optic projections. JONES immunoreactivity was briefly detected in coronal sections of the optic stalk at E13. It was present as a thin zone of label lying immediately ventral to the cell-free zone occupied by the first optic nerve axons and the flattened epithelia that will form the connective tissue of the optic nerve. At E16, JONES binding was detectable adjacent to the glial limiting membrane in the chiasm region and on ependyma and radial processes in ventral regions of the diencephalon. Immunoreactivity was particularly concentrated in the subpial re- 

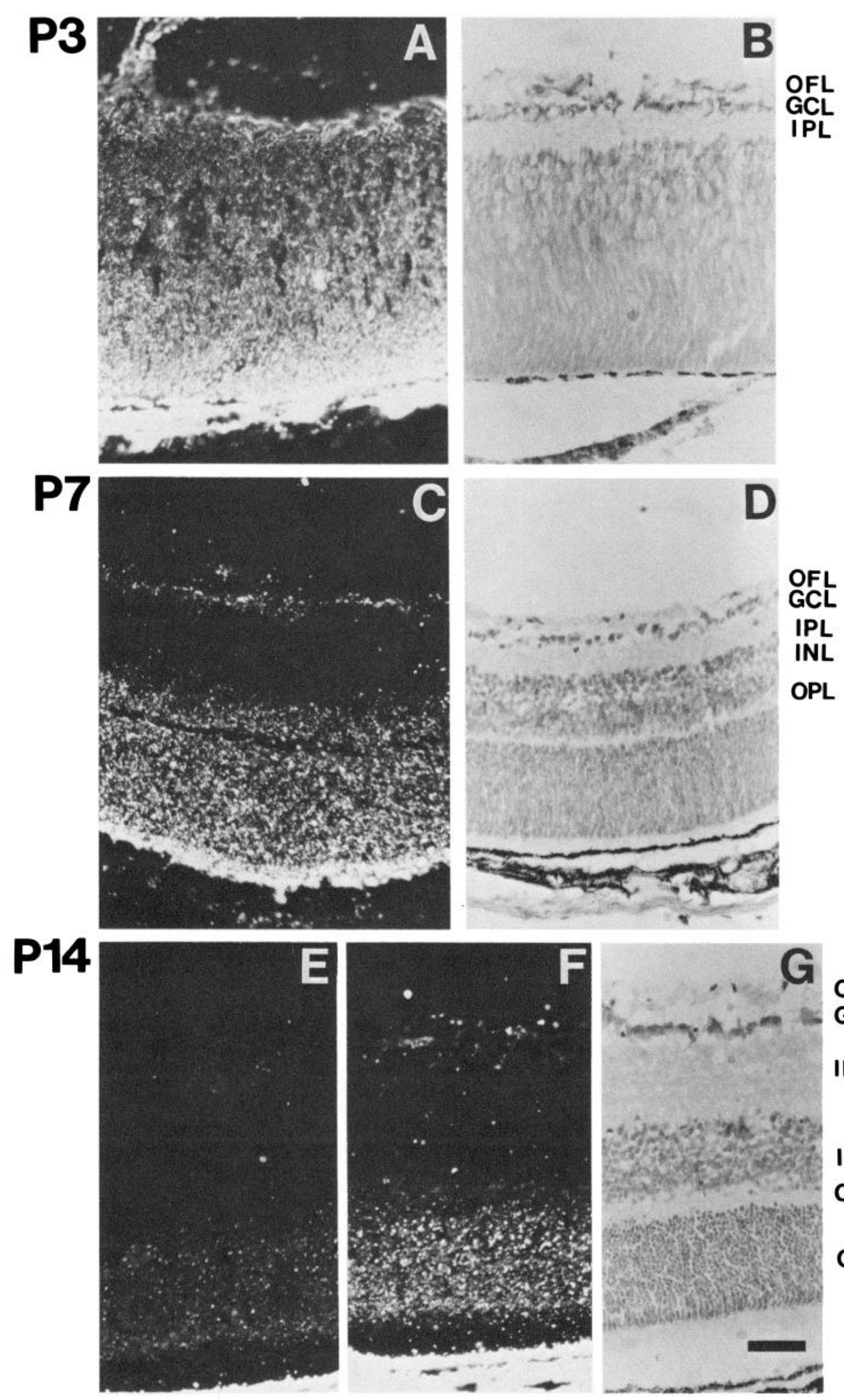

Figure 2. Pattern of JONES labeling in retinas from postnatal animals visualized with the immunogold procedure. Dark-field photomicrographs of immunogold-stained $(A, C, E, F)$ sections and bright-field photomicrographs of adjacent sections stained with toluidine blue $(B, D, G) . A$ and $B, \mathrm{P} 3$ retina: All retinal layers are stained with the JONES antibody, but the labeling in the inner layers is less intense than in the outer layers. $B$, Histological appearance of the retinas at this stage. The presumptive optic fiber layer $(O F L)$, ganglion cell layer $(G C L)$, and inner plexiform layer $(I P L)$ are marked. The other retinal layers are not yet present. $C$ and $D, P 7$ retina: JONES labeling has withdrawn from the inner plexiform INL layer, from the inner half of the deveOPL loping inner nuclear layer $(I N L)$, and OPL from the developing outer plexiform layer $(O P L)$. Labeling is apparent in the optic fiber layer. $E-G, \mathrm{P} 14$ retina:

ONL JONES staining is very weak in the central retina $(E)$, although it is still very intense in the periphery $(F)$. In this region the labeling is restricted to the developing outer nuclear layer $(O N L)$ and to the optic fiber layer. Scale bar, 50 $\mu \mathrm{m}$. gions adjacent to the developing optic tract (Fig. $3 A$ ). The optic tract can be clearly seen at E18 in parasagittal sections (Fig. $3 B$ ) stained with toluidine blue, and it is heavily labeled with JONES (Fig. $3 C$ ). The labeling drops abruptly in the next days, and by E20, the optic tract region is weakly labeled (Fig. $3 D$ ).

In the presumptive superior colliculus, JONES labeling is present in a radially oriented pattern that originates from the ventricular region. At E16, a superficial band of label can be detected under the pia in rostral lateral regions of the colliculus (Fig. 4A). Cell migration from the ventricular germinal zone of the colliculus occurs throughout the late fetal period (Bruckner et al., 1976). This is reflected in the intense periventricular la- 


\section{E16}

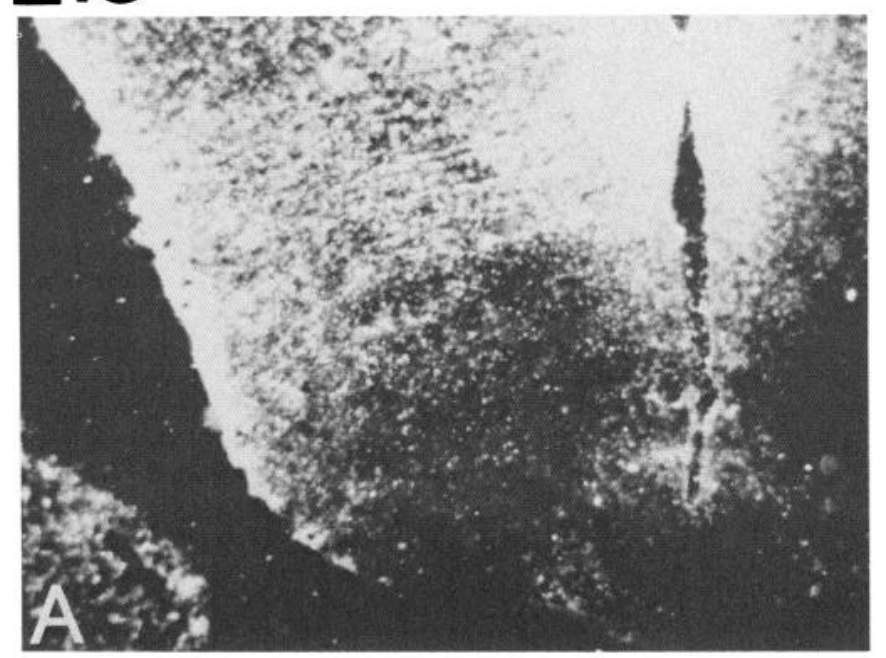

\section{E20}

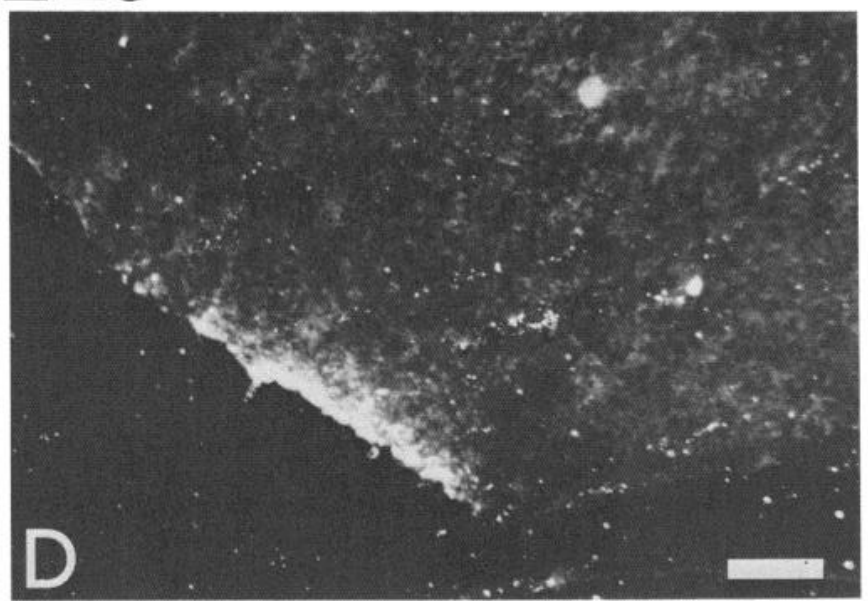

\section{E18}
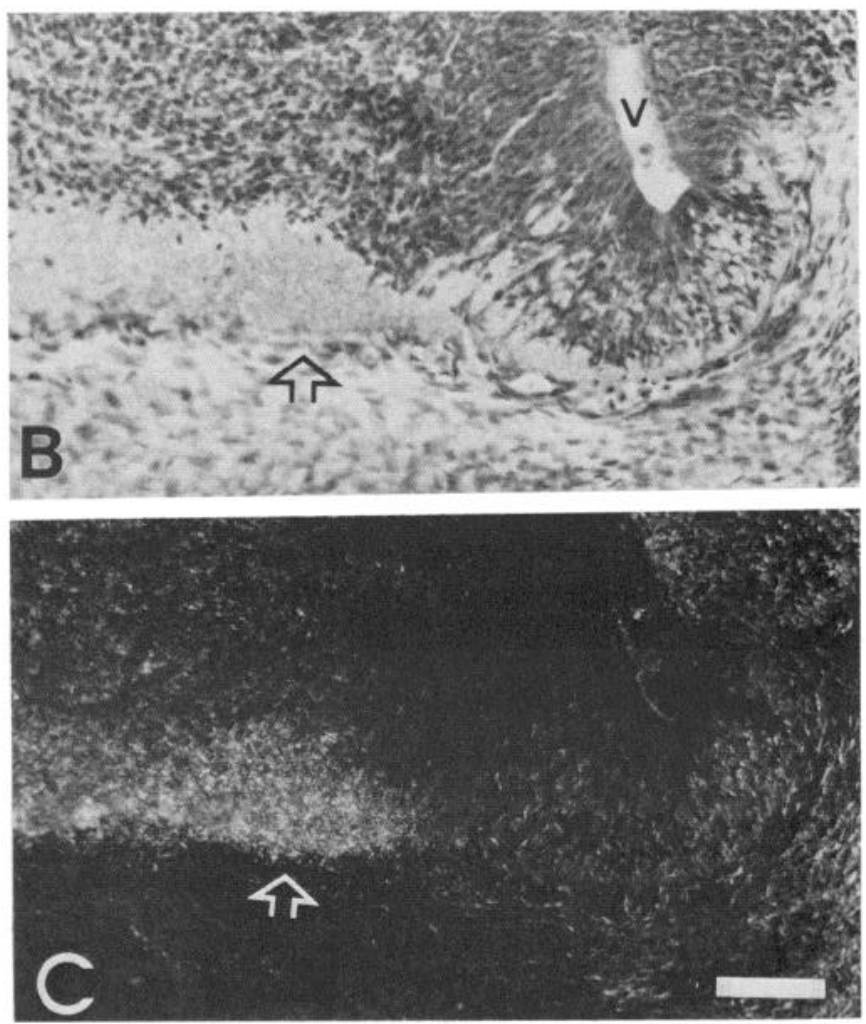

Figure 3. Pattern of JONES labeling along the developing optic pathway. A, Immunogold staining of a coronal section through the ventral diencephalon of an E16 animal. JONES antibody stains radially oriented process from the ventricle toward the diencephalic surface. The staining is much more intense in the lateral region of the diencephalon beneath the pia. $B$ and $C$, E18: Bright- and dark-field photomicrographs of a parasagittal section through the ventral diencephalon in an E18 animal. JONES binding was visualized wih the immunogold procedure $(C)$, and the section was counterstained with toluidine blue $(B)$. The intense JONES binding is localized to the position of the developing optic tract (arrow). The third ventricle is indicated $(V) . D$, E20: Dark-field photomicrograph of an immunogold-stained coronal section through the ventral diencephalon. JONES labeling is much more restricted in both the radial and subpial dimension, and, though difficult to discern in dark-field illumination, the intensity of the labeling that remains is reduced relative to the subpial labeling in the E16 brain. Scale bar: $A$ and $D, 100 \mu \mathrm{m} ; B$ and $C, 50 \mu \mathrm{m}$.

beling in caudal regions of the lobe. However, at E18, in addition to the intense ventricular staining, a band of intense immunoreactivity can be seen in rostral regions (Fig. $4 B$ ). This corresponds to a cell-free region in toluidine-stained sections (Fig. $4 C)$. By E19, antibody binding is distributed throughout the superficial collicular layers. However, after E19, there is a marked decrease in the immunoreactivity, and by P0, JONES binding has disappeared from the entire superior colliculus.

\section{Cerebellum}

In E14-18 fetuses there is a clearly defined cerebellar anlage that shows a pronounced pattern of JONES binding (Fig. 5, $A$, $B)$. At these ages, the labeling is present extending from the ventricular to the pial surface of the anlage. Most of this im- munoreactivity is distributed in a radially oriented pattern, with higher densities in caudal than in rostral regions. Immunoreactivity is also present, however, in the subpial region of the developing external granule cell layer, which, at this stage, begins its migration from the rhombic lip and is present as a densely packed zone of cell bodies in the caudal region of the anlage. A more rostral region of high-density JONES binding falls in a cell-free region between the pia and deeper cell somata (Fig. 5, arrows).

The cerebellum of the newborn rat is morphologically immature (Fig. 6B). The major fissures are recognizable, and the external granular layer (EGL) forms a sheet only a few cells thick. Parasagittal sections through the vermis reveal a difference in maturation between regions located rostral versus caudal 


\section{E16}

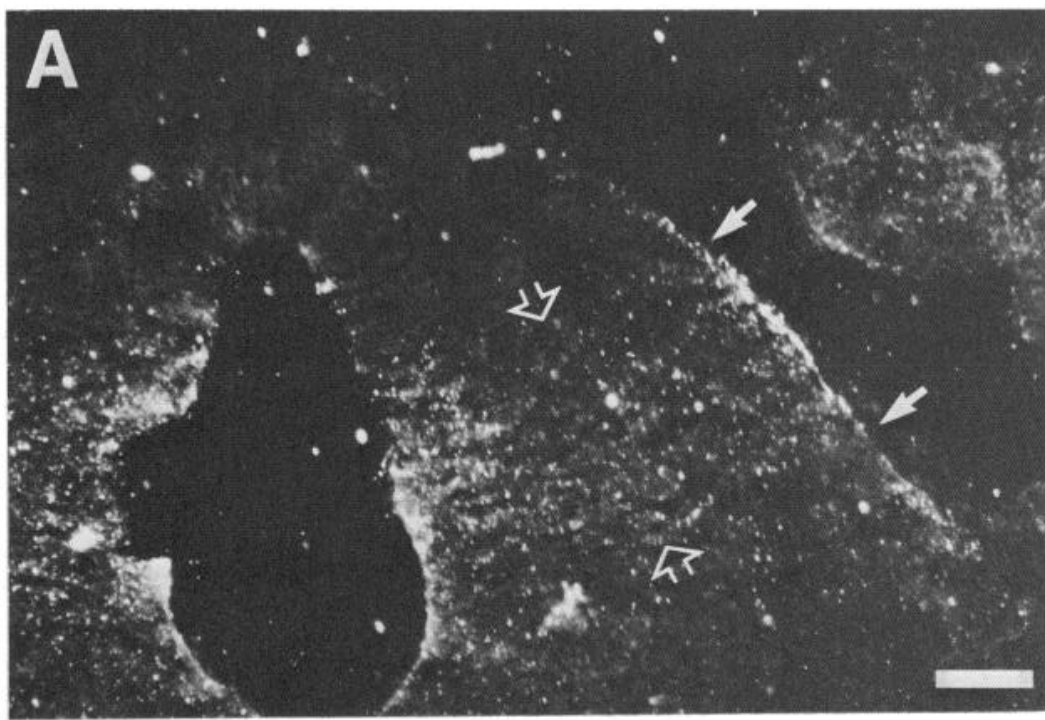

\section{E18}
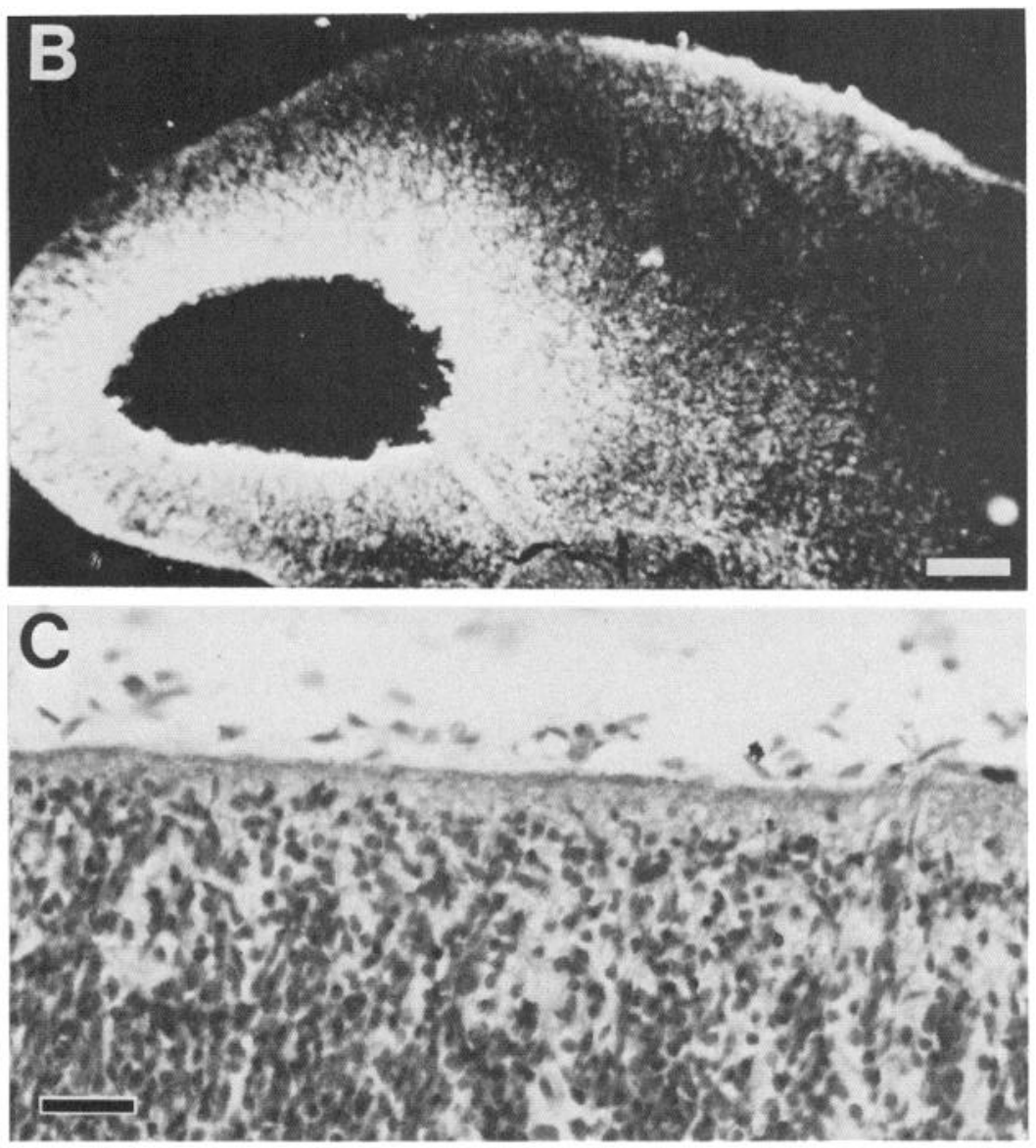

Figure 4. Pattern of JONES labeling in the presumptive superior colliculus. $A$, E16: Dark-field photomicrograph of an immunogold-stained coronal section through the dorsal mesencephalon. Observe labeling of processes radially oriented from the ventricle toward the tectal surface (open arrows) and also the intense labeling beneath the pia (closed arrows). $B$ and $C$, E18: Parasagittal section through the tectum stained with the immunogold procedure and counterstained with toluidine blue. Notice, in the dark-field photomicrograph $(B)$, the intense JONES labeling located beneath the pia in rostral regions of the midbrain. $C$, Detail of a section adjacent to the one shown in $B$ (bright-field optics). Note the cell-free region underneath the pia that shows heavy labeling in the dark-field optics. Rostral is to the left. Scale bar: $A$ and $B, 100$ $\mu \mathrm{m} ; C, 30 \mu \mathrm{m}$. to the fissura prima (Fig. $6 B$ ). In rostral regions of the cerebellar cortex, the Purkinje cell layer is becoming clearly defined and a few granule cells have settled below it. During the first postnatal days, JONES immunoreactivity exhibits a stereotyped but discontinuous pattern in the cerebellar cortex, which appears to correspond to differences in the maturity of different regions (Fig. 6A). In the most rostral regions, JONES binding is detected throughout the folium, with a radially oriented pattern extend- 


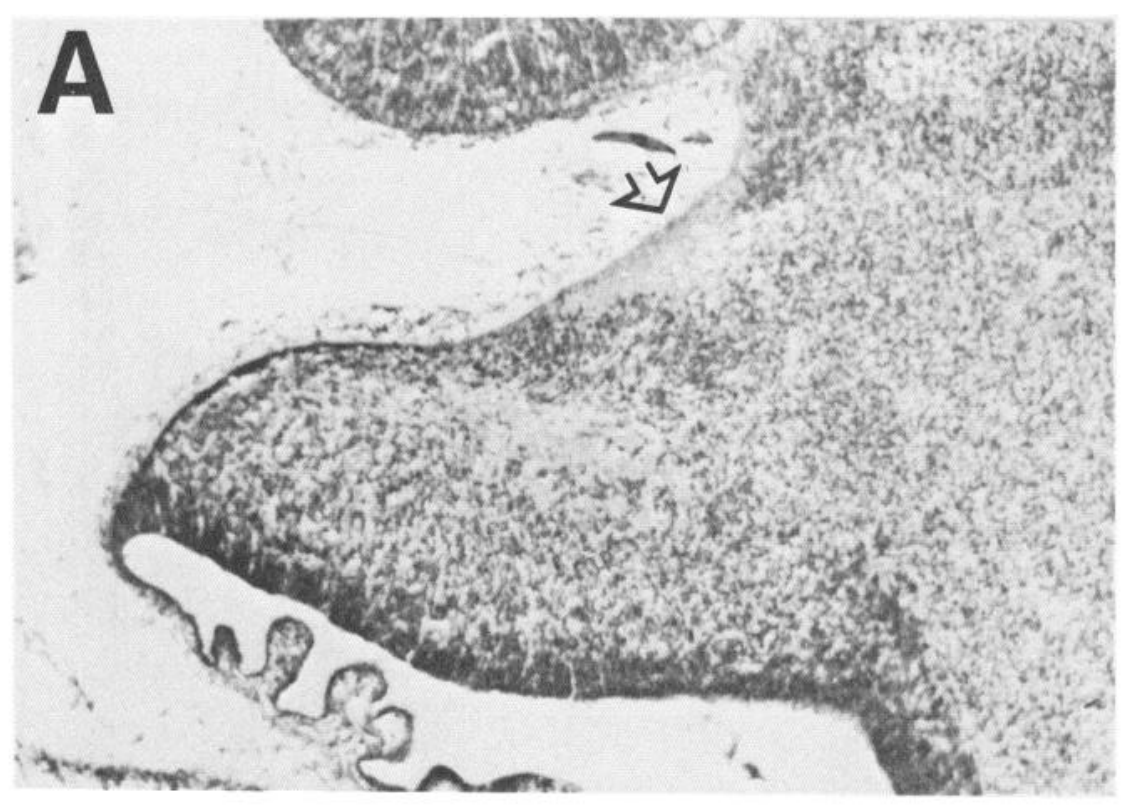

Figure 5. Distribution of JONES staining in the E18 cerebellar anlage. Immunogold staining of a parasagittal section counterstained with toluidine blue. Bright-field $(A)$ and dark-field $(B)$ photomicrographs of the same section illustrating the radially oriented pattern of JONES labeling along the dorsoventral axis of the cerebellar anlage. Dense subpial labeling in the caudaldorsal region of the cerebellar anlage corresponds to the region of granule cell migration from the rhombic lip. Observe also a rostrodorsal region of highdensity JONES binding corresponding to a cell-free region in the bright-field photomicrograph (arrows). Caudal is to the left. Scale bar, $100 \mu \mathrm{m}$.

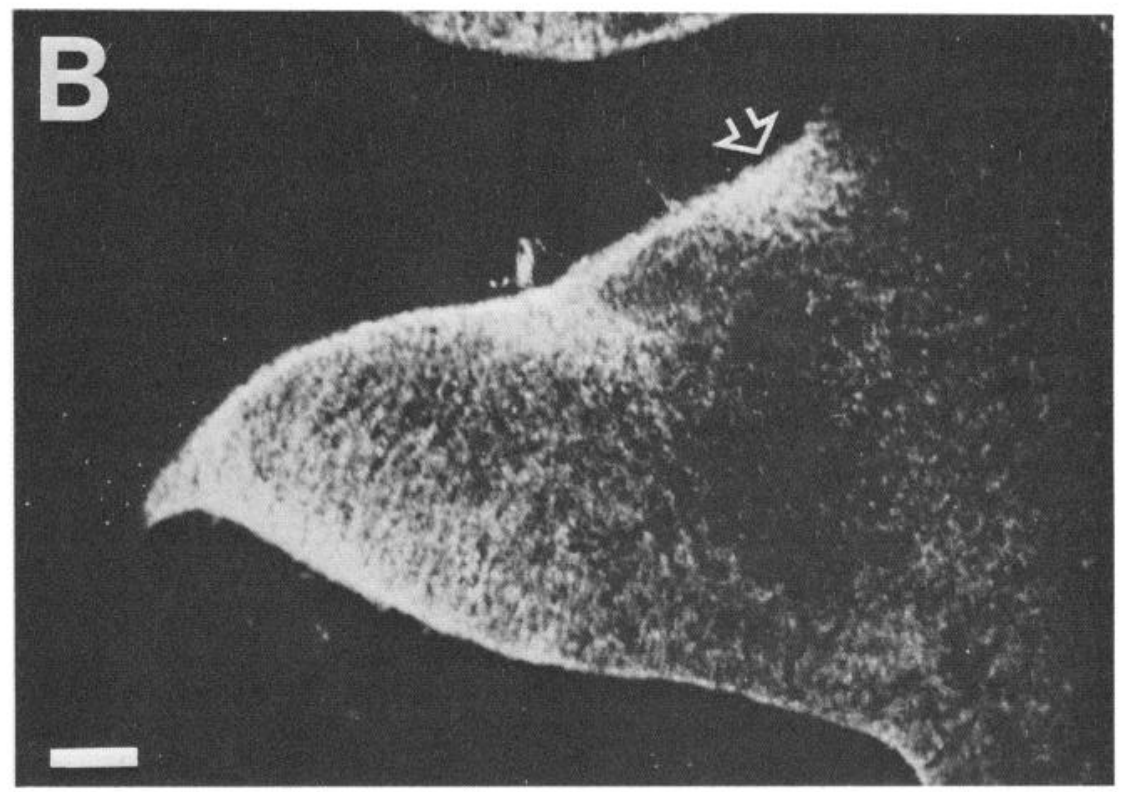

ing from the EGL and through the presumptive molecular layer. Caudal to the fissura prima, the presumptive cortical region is completely unstained.

By the end of the first postnatal week, the EGL is much thicker and the molecular, Purkinje cell, and granule cell layers can be delineated lying above the white matter in all regions of the cerebellar cortex (Fig. 7A). JONES staining of adjacent sections reveals labeling uniformly distributed throughout the cerebellar cortex, in regions corresponding to both the external granule cell layer and to the differentiating internal granule cell layer (Fig. $7 B$ ). The relative density of JONES binding at this stage corresponds roughly to the relative packing density of granule cell somata, being significantly higher in the external than in the internal granule cell layer. JONES immunoreactivity is markedly reduced in the deeper regions of the differentiating molecular layer, where parallel fiber projections are being laid down.
During the first postnatal week, a distinctly different pattern of JONES immunoreactivity was observed in the depth of the folia in the regions corresponding to the position of ingrowing fibers that will form the cerebellar white matter. In the neonate, intense JONES binding was present as patches and streaks distributed without any apparent order. By P7, JONES binding in the cerebellar white matter was distributed along the fiber fascicles of the differentiated white matter (Fig. $7 A$ ).

\section{Telencephalon}

The olfactory bulb and caudate are not yet clearly discernable in the rostral forebrain of the E13 rat, and the neocortex is represented by a thin, pseudostratified neuroepithelial sheet. By E16, the olfactory bulb is becoming discernable and the floor of the telencephalic ventricle has differentiated into the presumptive caudate, while dorsally the cerebral vesicles have expanded in thickness as well as in surface area. Intense JONES 

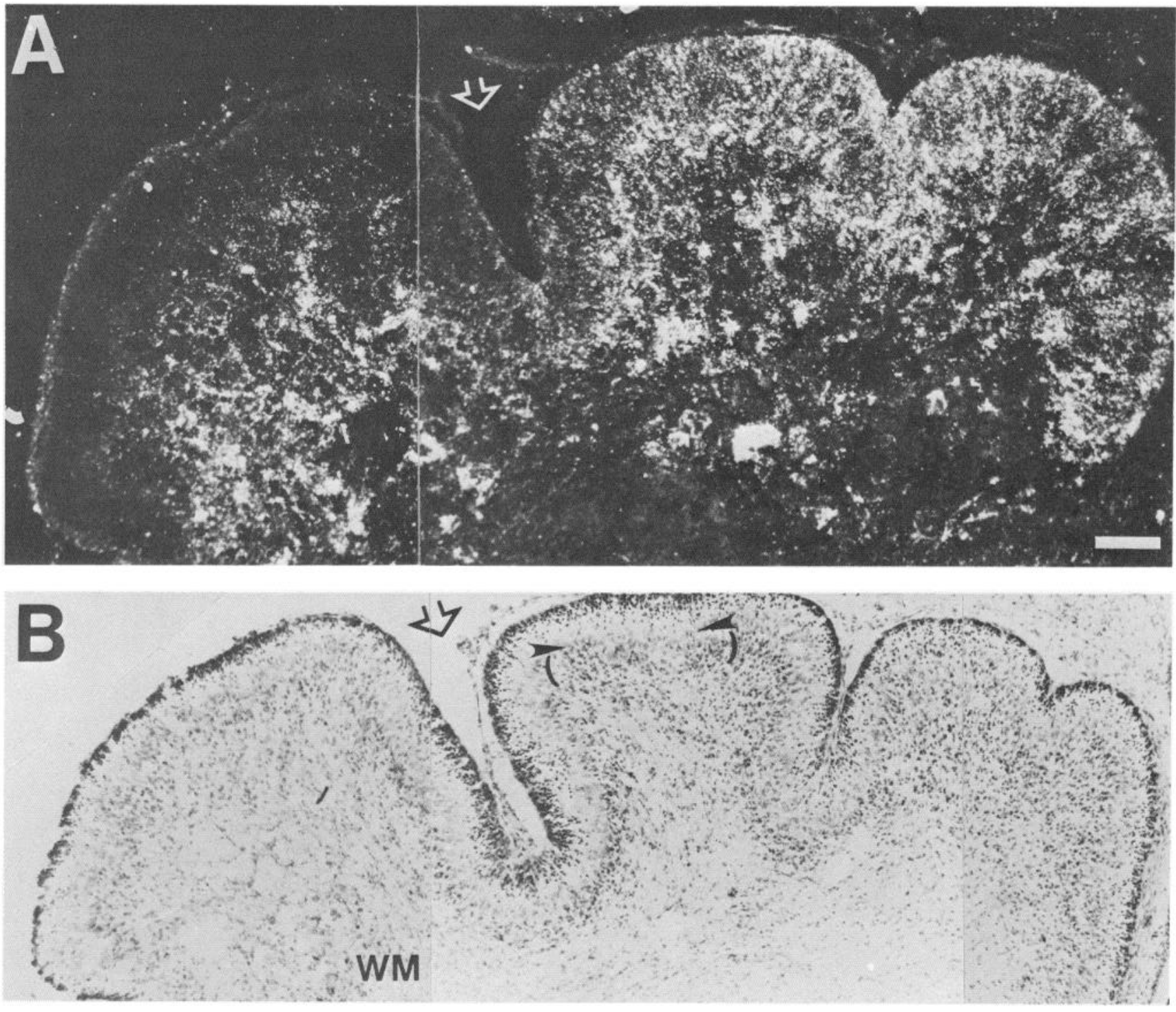

Figure 6. JONES expression in the early postnatal cerebellum. A, Dark-field photomicrograph of an immunogold-stained parasagittal section through the cerebellar vermis of a P0 animal. Rostral is to the left, and the arrow indicates the fissura prima. Observe JONES staining throughout the cerebellar cortex in the most anterior folia and the absence of labeling in the same region in the posterior folium. Patchy staining deep within the cerebellum corresponds to fascicles of axons within the developing white matter $(W M)$. $B$, Toluidine staining of a section through vermis to illustrate the cytoarchitectonic differences between both regions. The presumptive Purkinje cell layer (arrowheads) and granular layer (parentheses) are indicated. Scale bar, $100 \mu \mathrm{m}$.

immunoreactivity is present in the presumptive caudate region at this stage, as well as in the dorsal regions of the cerebral vesicles. In progressively older fetuses, as the cellular laminae began to form in the various neocortical regions, JONES immunoreactivity extends progressively toward the pia. In the E16 fetus, intense JONES antibody immunoreactivity is also observed extending from the olfactory epithelium to the olfactory bulb in regions where olfactory nerve bundles are localized.

By E18, the caudate remained intensely stained, and it was cleary delineated from adjacent regions of the telencephalic floor by the abrupt disappearance of JONES immunoreactivity (Fig. $8, A, B)$. In the cerebral vesicles themselves, there were both graded and abrupt changes in the intensity of JONES immunoreactivity across what otherwise appeared to be a continuous neural epithelium. One of the most abrupt transitions in JONES immunoreactivity can be observed in Figure $8 \mathrm{~A}$ (arrow), and apart from the abrupt differences in JONES binding, no other cytoarchitectonic differences were apparent in this region at this time.

At P0, JONES staining in the cortex shows a pattern reminiscent of Golgi staining of the radial glia at this stage (Fig. $8 \mathrm{C}$ ). Immunoreactivity has disappeared from the caudate and has withdrawn from the dorsal rostral regions of the neocortex at this stage. However, it is still intense in the ventricular zone of the cerebral vesicles and in the hippocampus, olfactory bulb, and olfactory nerve. At the end of the first postnatal week immunoreactivity was absent from the neocortex. It was still present, however, in the hippocampus, the olfactory bulb, and the olfactory nerve.

\section{Spinal cord and PNS}

JONES binding is first detected on E13 in the cervical region of the spinal cord and dorsal root ganglia. The labeling in these regions is not very intense relative to that of the brain. However, 

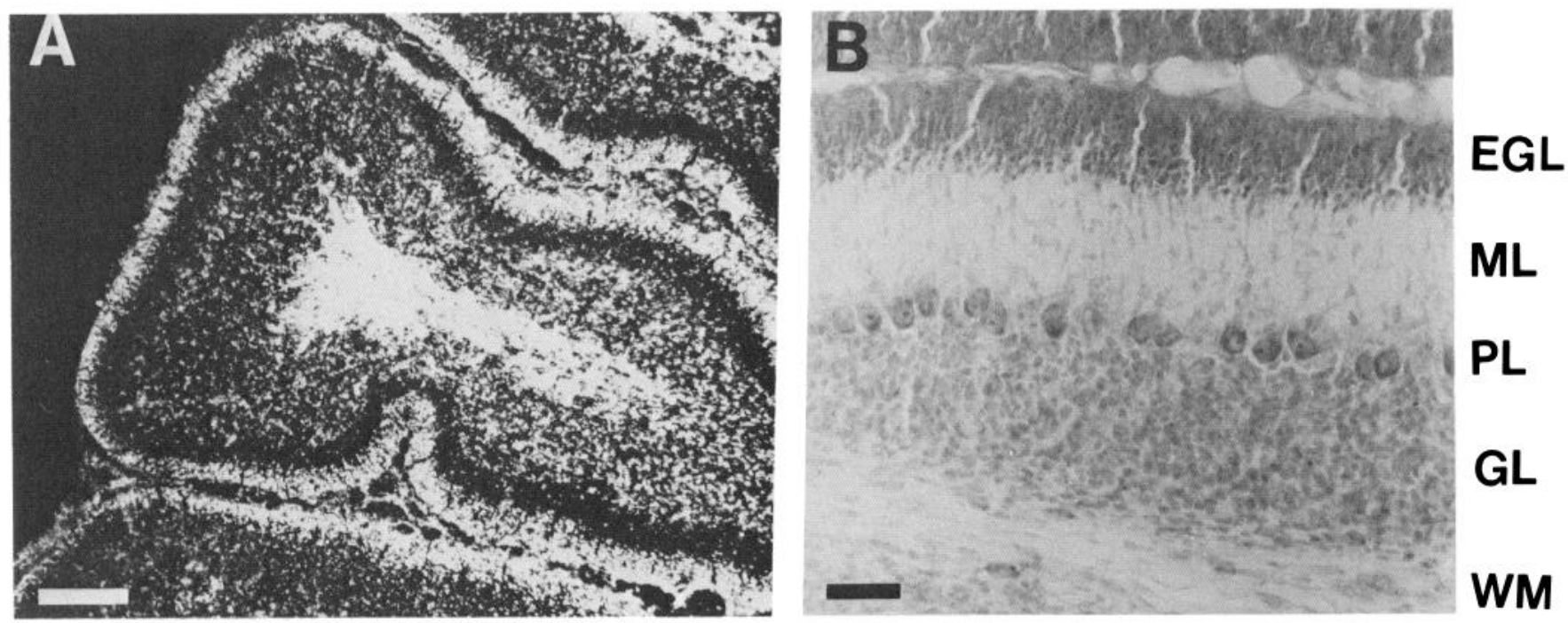

Figure 7. Distribution of JONES staining in the P7 cerebellum. A, Dark-field photomicrograph of an immunogold-stained parasagittal section showing JONES labeling throughout the cerebellar cortex and in the white matter. Observe the marked decrease in JONES labeling in the lower regions of the presumptive molecular layer. $B$. Histological appearance of the cerebellar cortex at this stage. The relatively cell-free zone in the lower regions of the presumptive molecular layer is the region where parallel fibers are developing. $E G L$, exernal granule cell layer; $M L$, presumptive molecular layer; $P L$, Purkinje cell layer; $G L$, internal granule cell layer; $W M$, white matter. Scale bar: $A, 100 \mu \mathrm{m} ; B, 50 \mu \mathrm{m}$.

immunoreactivity is heavily present in the dorsal root ganglia and in the dorsal root bifurcation zone by E16 (Fig. 9A). JONES binding is also present on radially oriented processes in the spinal cord that can be associated with radial glia present at this stage (Hockfield and McKay, 1985). JONES immunoreactivity is also present along the central and peripheral projections of the dorsal root ganglia and remains very intense in these regions until E18 (Fig. 9, $A, B$ ).

A detailed spatial and temporal description of the peripheral distribution of JONES antigen is beyond the scope of the present report. Nevertheless, even in the earliest fetus, JONES binding is heavily present in the trigeminal ganglia as well as in the peripheral and central trigeminal branches. The stained ramifications of the trigeminal system can be followed in the periphery, and the innervation of individual vibrassae can be resolved. However, at no embryonic or postnatal stage have we observed binding of the antibody in association with any peripheral somatic motor nerves or innervation fields.

By E20, JONES immunoreactivity has almost disappeared from the spinal cord and dorsal root ganglia, as well as from the trigeminal system.

JONES immunoreactivity has been observed in only 2 "nonneural" regions: It is present in a punctate pattern in the glomeruli of postnatal and adult kidneys and in the adrenal medulla at the same stages. The latter staining may be associated with autonomic innervation, but we have not pursued this question.

\section{Adult nervous system}

By P60, JONES binding shows the adult pattern of staining in all regions of the brain. It is immunocytochemically detected only in the retina and cerebellum. In the retina, the antigen shows a punctate pattern in the outer plexiform layer and a discontinuous distribution in the optic fiber layer (Fig. 10). The labeling in the optic fiber layer extends to the optic nerve head, where it is very intense and continuous, but it stops abruptly outside the orbit (Fig. 10D). In the cerebellum, the antigen shows a radially oriented pattern throughout the molecular layer and delineates cell bodies in the Purkinje cell layer (Fig. 11).

\section{Comparison with D.1.1 antibody}

A ganglioside antigen containing an $\mathrm{O}$-acetylated sialic acid residue has been characterized using the D.1.1 antibody in the developing rat nervous system (Cheresh et al., 1984; Levine et al., 1984). Recent studies demonstrated that at least one of the bands recognized by the JONES antibody on HPTLC plates is a base-sensitive O-acetylated derivative of GD3 (Blum and Barnstable, 1986), suggesting that both antibodies might recognize the same ganglioside species. The antigen recognized by the D.1.1 antibody has been localized exclusively to germinal cells in the developing rat CNS (Stallcup et al., 1983; Levine et al., 1984; Levine et al., 1986; Beasley and Stallcup, 1987). We therefore felt it important to compare the binding of these 2 monoclonal antibodies on similar tissue sections. Adjacent tissue sections from $\mathrm{P} 0$ forebrain were reacted with both antibodies as described under Material and Methods. Figure 12 shows that both JONES (Fig. 12A) and D.1.1 antibody (Fig. 12B) label the ventricular zone and also radially oriented processes throughout the intermediate zone. We also stained tissue sections from P6 cerebellum, and the staining pattern with D.1.1 was similar to that described with JONES. It thus appears that both antibodies recognize antigens with similar distributions and that this distribution is not limited to regions of cell proliferation.

\section{Discussion}

Antibody technology provides a powerful tool in bridging the experimental and conceptual gaps between descriptions of brain development and their underlying molecular mechanisms. In the case of an antibody such as JONES, raised against a crude tissue homogenate, immunocytochemistry is a crucial first step in deciding whether the antigen or antigens are of sufficient developmental interest to warrant further effort. Consequently, in the work reported above we have attempted to provide an 

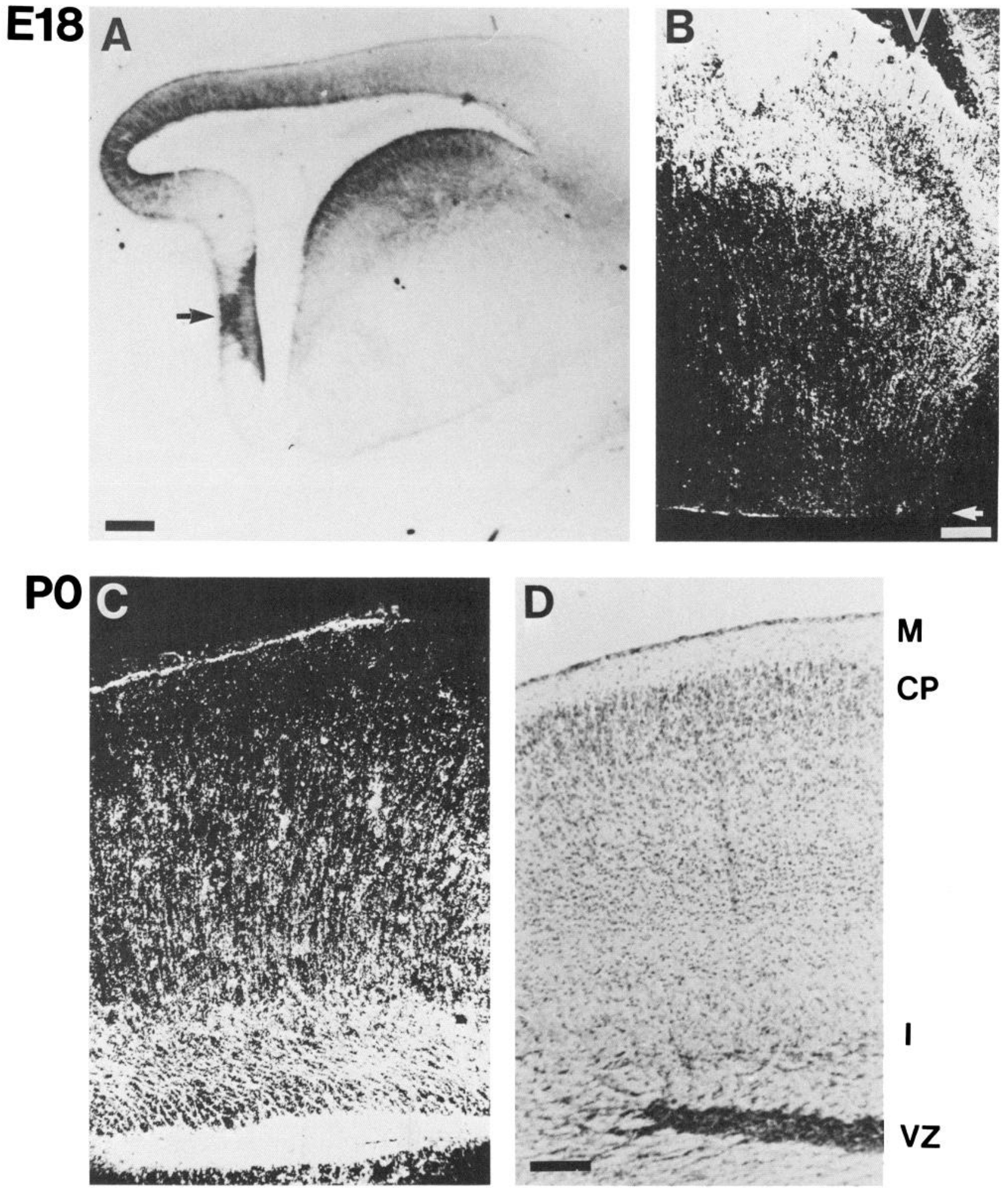

Figure 8. Pattern of JONES labeling in the embryonic and postnatal telencephalon. A, Bright-field photomicrograph of an immunogold-stained parasagittal section of an E18 animal. Observe JONES staining in the presumptive caudate and the abrupt change in the distribution of JONES staining in the caudal part of the cerebral cortex anlage (arrow). B. High-power view (dark-field optics) of the same section illustrated in $A$ to show the pattern of labeling in the ventral telencephalon. Intense labeling is present near the ventricle $(V)$ with radially oriented processes ending in the ventral pia (arrow). $C$, Immunogold staining (dark-field optics) to show the pattern of JONES labeling in parasagittal sections through the occipital cortex of a P0 animal. Note the intense staining in the ventricular region and the labeled processes that originate in the ventricular region and end in the pia. The pattern is highly reminiscent of Golgi images of radial glia at this stage. $D$, Toluidine staining of an adjacent section to show the histological appearance of the occipital cortex at this stage. $M$, marginal zone; $C P$, cortical plate; $I$, intermediate zone; $V Z$, ventricular zone. In all the panels, dorsal is $u p$ and caudal to the left. Scale bar: $A, 100 \mu \mathrm{m} ; B, 50 \mu \mathrm{m} ; C$ and $D, 100 \mu \mathrm{m}$. 


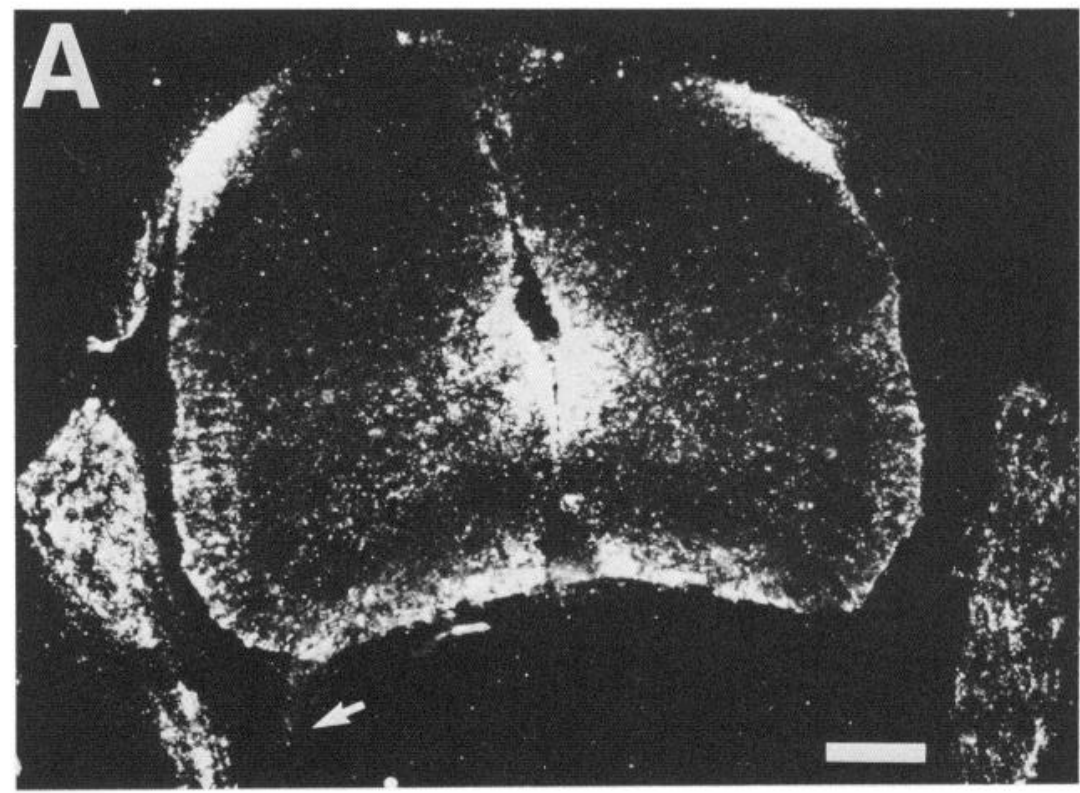

Figure 9. Pattern of JONES binding in the spinal cord and dorsal root ganglia. $A$, Dark-field photomicrograph of a coronal section through the cervical spinal cord of an E16 rat. JONES immunoreactivity is apparent in the dorsal root ganglia, in the central and peripheral roots, and in the dorsal funiculus of the spinal cord. Labeling is also present as radially oriented processes from the central canal toward the pial surface. At this stage, relay neurons are still migrating to the lateral border of the gray matter, and their axons are growing to form the lateral funiculus (Altman and Bayer, 1984). The ventral root (arrow) is unstained. B, Parasagittal section through cervical dorsal root ganglia at E18 stained with the immunogold procedure. Peripheral nerves are to the right. The spinal cord is not present in this section. Scale bar ( $A$ and $B), 50 \mu \mathrm{m}$.

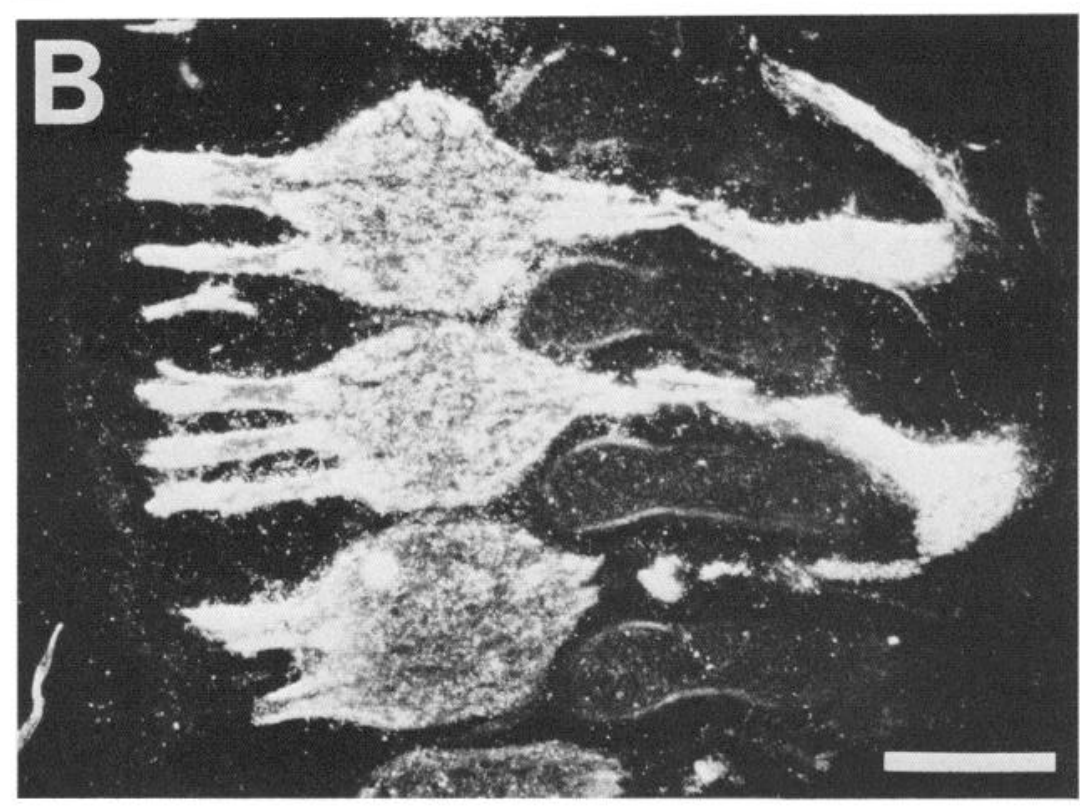

overview of the spatially and temporally varied binding distribution of this mab in the developing rat nervous system. At the same time, we have paid particular attention to those regions for which earlier work provides descriptive background sufficient to test the hypothesis that the JONES antigens are invariably associated in both position and timing of their appearance with cell and axon migrations. The observations presented in this report strongly support the hypothesis we set out to test. In all regions we have examined, JONES binding localizes in both position and time to migrating cell populations or to developing axon projections.

\section{Correlation with cell migrations}

The rat retina, like that of most other mammals, differentiates in a central to peripheral order across its surface and in an inner to outer sequence across its radial dimension (Weidman and Kuwabara, 1968; Braekevelt and Hollenberg, 1970; Kuwabara and Weidman, 1974; Raedler and Sievers, 1975). Thus, in the early fetal stages, only the most central regions of the retinal epithelium are producing the postmitotic neuroblasts that begin migrating toward the vitreal surface to eventually become mature ganglion cells (Maslim et al., 1986). This zone of newly generated migratory neuroblasts gradually enlarges to reach the ciliary margin of the retina by birth. We have reported earlier that the tangential distribution of JONES binding in fetal retina closely parallels the peripheral expansion of the zone of migratory neuroblasts (Constantine-Paton et al., 1986). In the present study we have followed the distribution of JONES binding across the tangential and radial dimensions of the retina during the postnatal period. At these stages we have found, in accord with our hypothesis, that the gradual withdrawal of JONES immunoreactivity follows the central to peripheral and inner to outer sequence of cessation of migratory activity. Thus, for the tangential dimension, in the fetus, JONES binding appears first in central retina and then gradually spreads to the retinal margin. By contrast, in the postnatal period, JONES immunoreactivity 

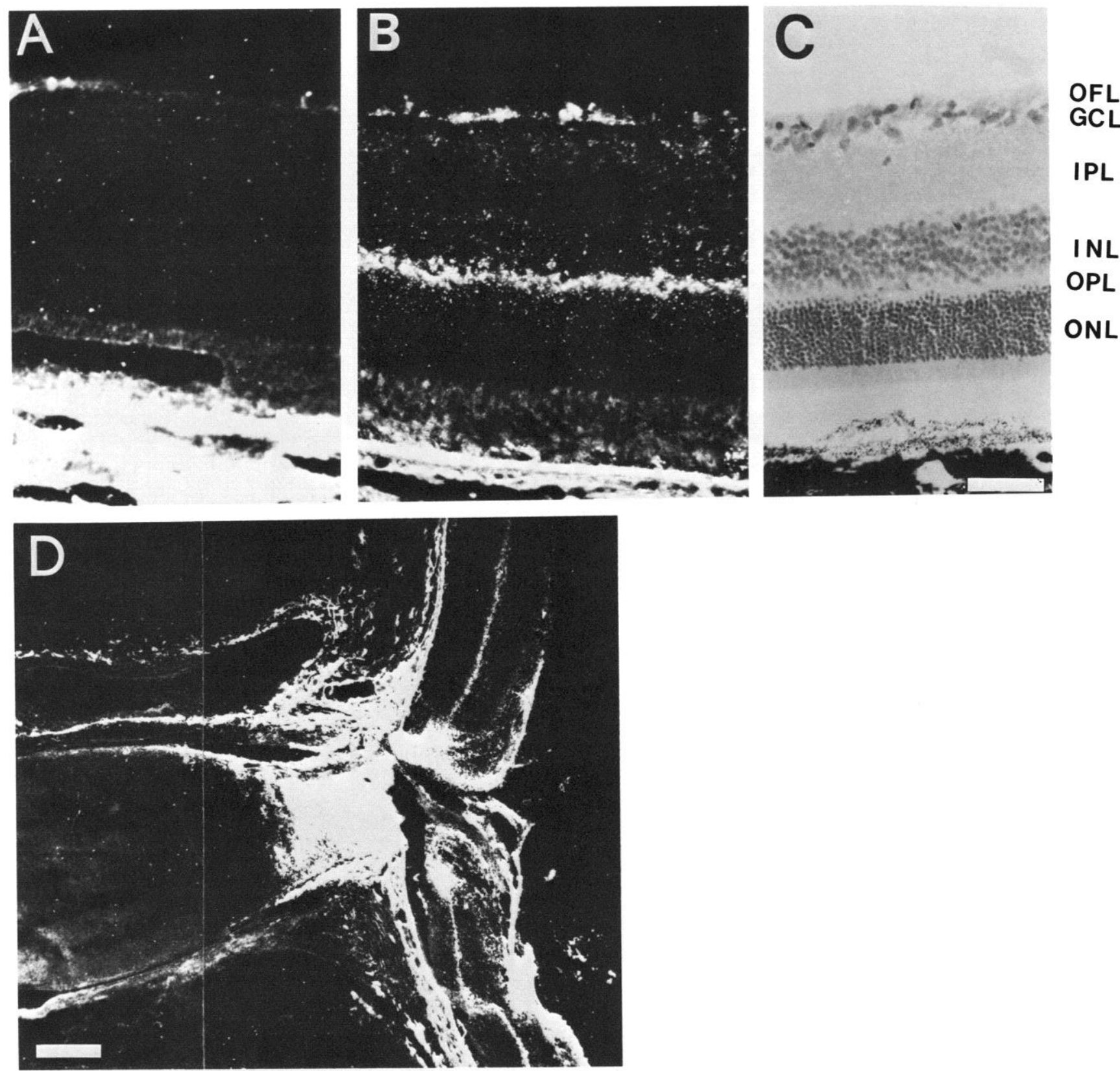

Figure 10. JONES staining in the adult retina and optic nerve head. $A$ and $B$, Dark-field photomicrographs of sections through the retina incubated with JONES antibody $(B)$ or with goat serum $(A)$ followed by immunogold staining. In $B$ note the JONES labeling in the optic fiber and outer plexiform layers. $C$, Histological appearance in an adjacent section stained with toluidine blue. Retinal layers are marked: optic fiber layer ( $O F L$ ), ganglion cell layer $(G C L)$, inner plexiform layer $(I P L)$, inner nuclear layer $(I N L)$, outer plexiform layer $(O P L)$, and outer nuclear layer $(O N L)$. Scale $\operatorname{bar}(A-C), 50 \mu \mathrm{m}$. $D$, Section of retina at the point of emergence of the optic nerve, stained with the JONES antibody (dark-field optics). Observe the heavy staining of the optic nerve head, and the absence of staining immediately after the nerve exits the orbit. Scale bar, $100 \mu \mathrm{m}$.

fades to near background levels first in central retina (Fig. 2E) at the time its mature lamination pattern is complete (Braekevelt and Hollenberg, 1970). Several days later, when all retinal cell migrations have ceased, the same low level of immunoreactivity is found near the ciliary margin.

The same correlation is present along the radial retinal dimension. At birth, in central retina only the last generated ganglion cells and some displaced amacrine cells are still migrating through the discernible inner plexiform layer, while in the inner retina the majority of inner nuclear layer cells are in the process of migration. In accord with this radial difference in the amount of migratory activity, JONES immunoreactivity in PO retina is still extremely heavy in the outer retina. However, binding of the antibody has significantly decreased in the inner plexiform and ganglion cell layer regions (Fig. $2 A$ ). In progressively older postnatal animals, immunoreactivity disappears successively in the ganglion cell, inner plexiform, and inner nuclear layers. At P14, JONES binding is significantly decreased in magnitude and is restricted to the differentiating photoreceptor cell bodies (Fig. $2 F)$.

Cerebellar differentiation involves 3 distinct patterns of cell migration (Altman, 1969; Altman and Bayer, 1985a-c). Prenatally, Purkinje cells, Golgi cells, and deep nuclear neurons originate in the ventricular zone and migrate into the body of 

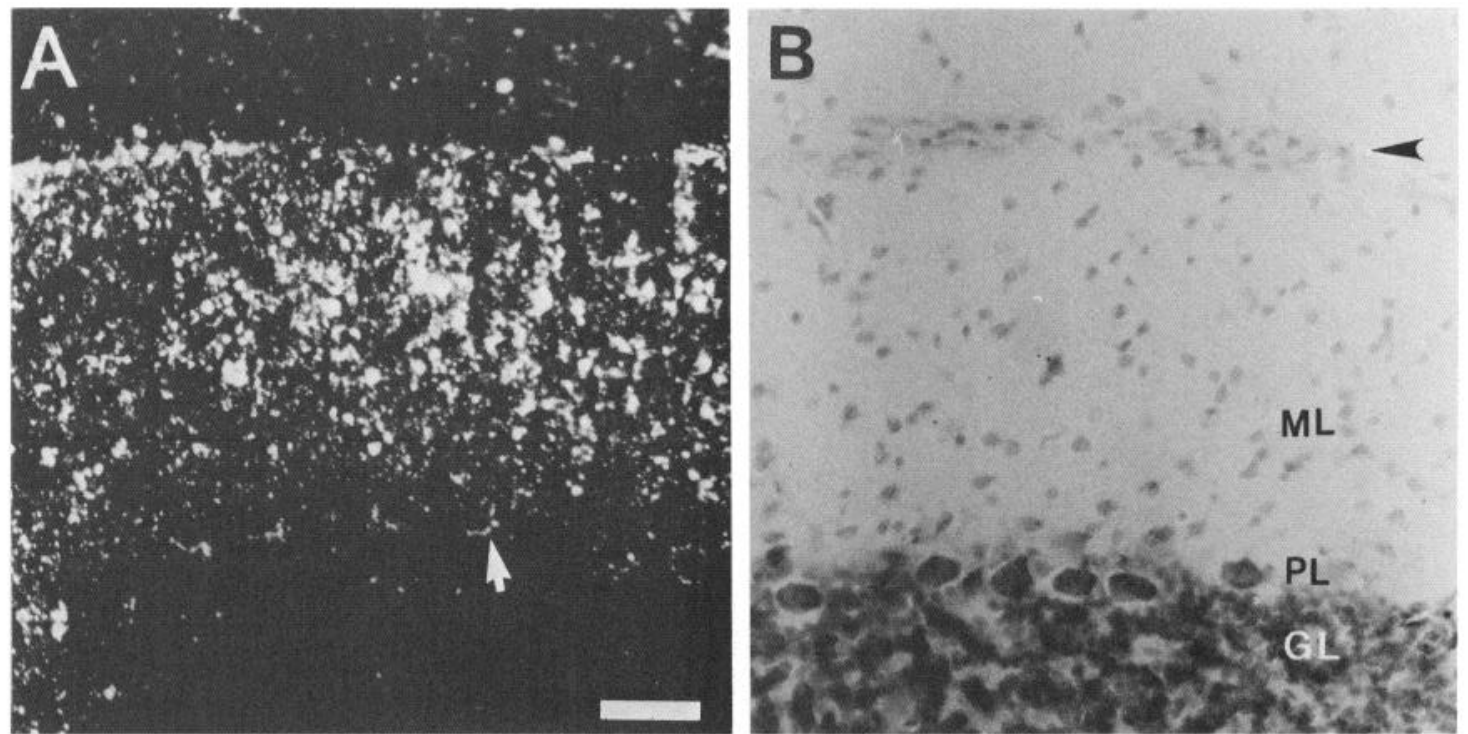

Figure 11. Pattern of JONES labeling in the adult cerebellum. A, Dark-field photomicrograph of a parasagittal section through the cerebellar cortex stained with immunogold. Intense labeling extends throughout the molecular layer and around the Purkinje cell bodies (arrow). JONES immunoreactivity is absent from the granular layer and the white matter. $B$, Light-field photomicrograph of an adjacent section stained with toluidine blue. Arrowhead points to the pia, and the cerebellar cortical layers are marked: molecular layer $(M L)$, Purkinje layer $(P L)$, and granular layer $(G L)$. Scale bar, $50 \mu \mathrm{m}$.

the cerebellar anlage. This is a radial migration from the ependymal region toward the pia. At E18, the positions of these dorsally migrating cells correspond closely to the radial pattern of JONES staining extending from the ependymal border upward through the anlage (Fig. $5 B$ ). The primary tangential mi- gration of cells destined for the external germinal zone, or the EGL, is also underway in the cerebellum at this stage. This migration can be observed as a thin layer of densely stained cells in the subpial region in the caudal half of the anlage, and it colocalizes with a subpial strip of JONES labeling (Fig. $5 B$ ).
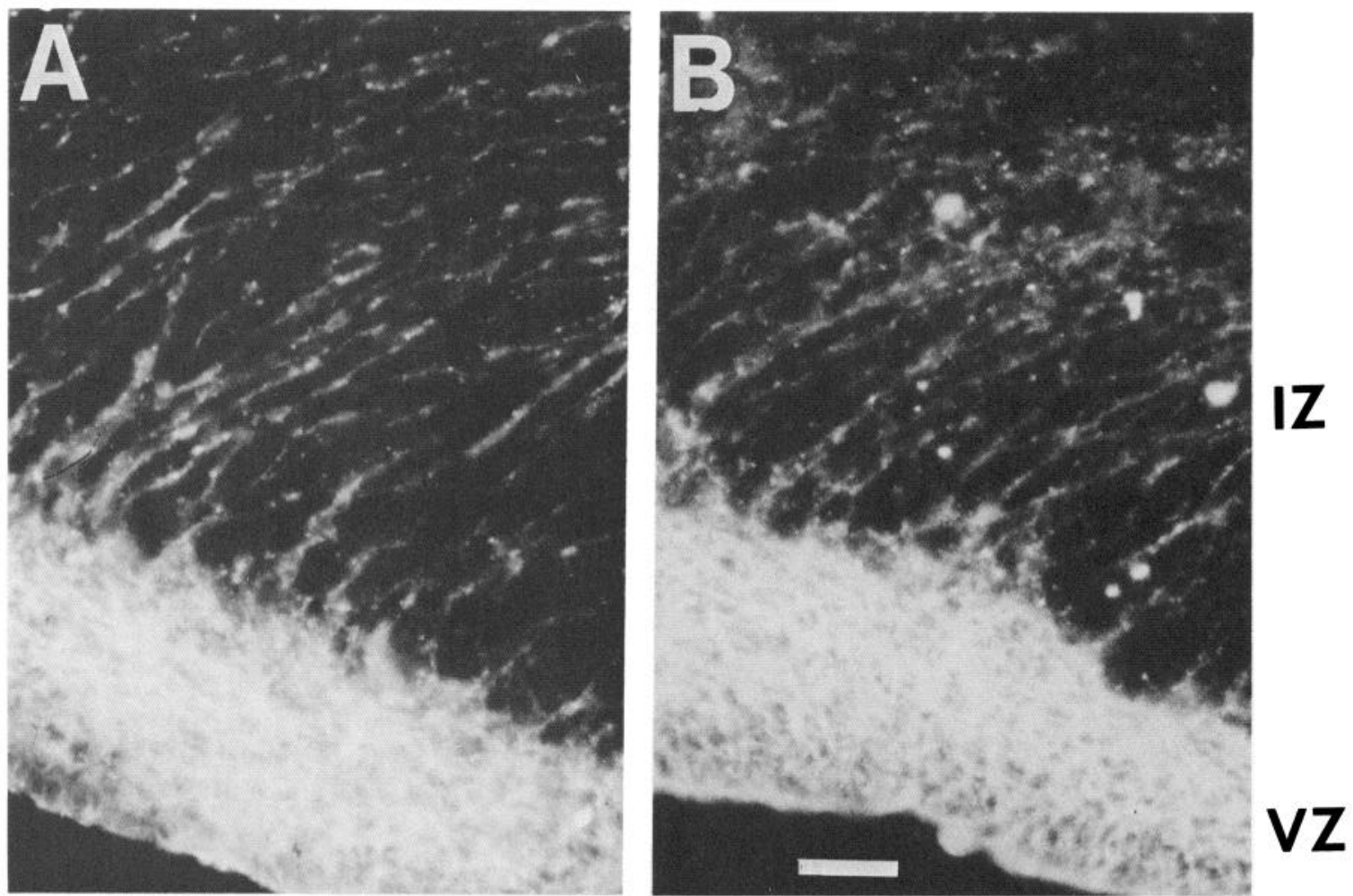

Figure 12. Comparison with D.1.1 antibody. Adjacent parasagittal sections through the occipital cortex in a P0 animal stained with JONES (A) or with anti-D.1.1 antibody $(B)$. Observe the labeling of the ventricular zone and also of the processes radially oriented toward the pial surface with both antibodies. $V Z$, ventricular zone; $I Z$, intermediate zone. Scale bar, $50 \mu \mathrm{m}$. 
In the postnatal period, the EGL gives rise to several types of neurons but predominantly to the granule cells which migrate inward from the pial surface into the future granular layer. This postnatal migration of granule cells has been shown to follow the processes of Bergman glia (Rakic, 1971) and to occur in different subdivisions of the cerebellar cortex at different ages (Altman, 1969). For example, in the vermis, the ventral lobes mature before the anterior lobes, and the last maturing folia are those located caudal to the fissura prima. At P0, JONES immunoreactivity is absent in the posterior lobes of the vermis, where EGL cell proliferation is pronounced but where cell migration has not yet taken place. JONES labeling oriented in radial arrays through the presumptive molecular layer becomes apparent, however, in regions located anterior to the fissura prima, where the inward migration of granule cells is already underway (Fig. 6A). By P7, granulc cell migration is well underway throughout the cerebellar cortex, the internal granule cell layer has begun to form as granule cells migrate to positions below the Purkinje cells, and the first parallel fiber bundles form a tightly packed fiber layer between the EGL and the Purkinje cell layer. In the P7 cerebellum, JONES immunoreactivity extends throughout the cerebellar cortex, where its binding is coextensive with the migrating granule cells and in roughly the same proportion to the density of these cells (Fig. 7, $A, B$ ). The differential expression of JONES antigen in the postnatal cerebellar cortex can therefore be related to the regional and temporal differences in the migratory patterns of the developing granule cells.

Similar correlations between JONES localization and cell migration are clear in our less-detailed examinations of the telencephalon and spinal cord. Thus, when JONES binding is first visualized (at approximately E12), the first postmitotic neurons are being generated (Hicks and D'Amato, 1968; Raedler and Sievers, 1975, 1976; Bruckner et al.. 1976; Lund and Mustari, 1977). In older animals, JONES is present in the ventricular, subventricular and intermediate zones and more lightly in the cortical plate and marginal zone of the differentiating neocortex. By birth, when the last neurons generated are still migrating to form the superficial layers (Hicks and D'Amato, 1968), JONES binding shows a pronounced radial pattern that appears to delineate the radial glia, presumed substrates for this migration (Rakic, 1971). In the telencephalon, as in the retina and cerebellum, the disappearance of JONES immunoreactivity also appears to be correlated with cessation of cell migration. Thus, JONES binding is heavy in the caudate throughout the fetal period but has disappeared by birth. At P0, the only telencephalic regions that continue to express JONES are the late differentiating zones: the occipital cortex, the hippocampus, and the olfactory bulb. By P7, only olfactory bulb and hippocampus show low levels of the JONES binding, and during the second postnatal week, as cell migrations cease in these late differentiating areas, JONES immunoreactivity also disappears (Altman and Das, 1965; Altman, 1966). A correlation with cell migration may also underly JONES labeling in the gray matter of the spinal cord seen in Figure $9 A$. Even though most lateral motor column migration has occurred by this stage, the labeling can represent the migration of ipsilaterally and contralaterally projecting relay neurons in this region (Altman and Bayer, 1984).

\section{Correlation with axon growth}

We first became interested in the JONES mab because its binding pattern showed a graded topographic distribution across the retinal epithelium and the antigenicity could also be localized in the optic fiber layer of embryonic and early postnatal retina (Constantine-Paton et al., 1986). This suggested that the JONES antigen could play a role in the selective pathway choices made by the growing retinal ganglion cell axons. Our present observations on the differentiating optic pathway are consistent with this possibility. JONES immunoreactivity colocalizes exceptionally well with the positions where retinal ganglion cell axons grow and with the time the major wave of growth cones are in a particular region of the pathway. For example, we have seen it only briefly in the optic stalk of the E13 animals (not shown), when the first axons exit the eye primordia (Horsburgh and Sefton, 1986). Immunoreactivity appears in the chiasm around this time, and over the next few days, JONES binding increases dramatically in the diencephalon, particularly in the subpial regions occupied by the optic tract (Silver and Sapiro, 1981). The subpial localization of JONES immunoreactivity in the rostrolateral regions of the colliculus at E18 (Fig. $4 B$ ) also corresponds to the position of retinal axons in this structure at this stage (Lund and Bunt, 1976; Bunt et al., 1983). By E20, when the major wave of retinal axon growth cones has traversed the diencephalon, JONES immunoreactivity has dramatically decreased in this region (Fig. 3D). By birth, when all but the youngest retinal ganglion cells have arrived in the superior colliculus, JONES immunoreactivity has disappeared from all the thalamic and midbrain areas receiving direct retinal projections.

Our observations of the developing optic tract are not yet at a sufficient level of resolution to either rule out or support the further possibility that the differential distribution of JONES immunoreactivity in the retina is mirrored by a complementary distribution of reactivity along the pathway and in the colliculus.

In numerous other regions of the developing rat nervous system JONES binding colocalized with differentiating axon tracts and appeared to be roughly related in amount and timing of reactivity to periods when the largest numbers of axons would be growing. This occurs, for example, in the dorsal root ganglia, the dorsal funiculus, the peripheral sensory nerves, the olfactory nerves, and the white matter of the cerebellum. We have not, however, studied any pathways with techniques that would allow fine distinctions between which subregion of a compact axon tract is showing the most intense JONES immunoreactivity. In fact, the intensified immunogold reaction tends to saturate completely in most pathway regions and is therefore not suited for a study of this type.

It is important to point out that, in this comparison of JONES binding and cell and axon migrations, we have emphasized and indeed studied only those pathways and brain regions where JONES immunoreactivity is present. There are likely to be other pathways and instances of cell migration, such as the peripheral motor nerves or ventral retina (Constantine-Paton et al., 1986), which appear to complete differentiation without ever expressing the JONES antigens or which express them at low levels that are not proportional to the amount of migratory activity likely to be underway. This differential expression of JONES binding within the migratory pathways of the devcloping brain suggests that the JONES antigens function to modulate some process involved in migration rather than serving a crucial role in the mechanism of motility itself.

The absence of definitive information on the cell types that express JONES should also be noted. In the diencephalon, cortex, cerebellum, and spinal cord, the binding appears to be coextensive with the radial glia. In the peripheral and cranial ganglia, 
however, it appears to be on the neurons and the axons themselves. We therefore suspect that JONES does not bind to a particular cell type but rather serves to delineate the substrate that is being used for a particular migratory route. This may mean that the expression of the JONES antigens occurs independently on a particular substrate or that its expression is induced as a result of the interaction between particular migratory cell membranes and particular substrates. Such questions can only be resolved in future studies using in vitro assays, other antibodies, and the electron microscope.

\section{Lack of correlation between JONES immunoreactivity and cell division}

In the following paper, we present evidence that the JONES antibody recognizes the same 2 to 3 gangliosides in all the regions studied in the present report. These are probably di- or trisialogangliosides (Schlosshauer et al., 1988). Disialogangliosides have previously been localized in proliferative zones (Stallcup et al., 1983; Goldman et al., 1984; Levine et al., 1984, 1986; Beasley and Stallcup, 1987), and since in many brain regions cell proliferation overlaps with cell migration, it is important to rule out the possibility that the JONES antigens are associated with cell division per se. There are 3 observations that argue against this possibility. First, the initial appearance of JONES immunoreactivity at E11.5-E12 is closely correlated with the first appearance of postmitotic cells in the fetal rat brain (E12), as reported by earlier investigators (Raedler and Sievers, 1975). In the earlier stages of fetal development, intense mitotic activity is present throughout the neural tube yet no JONES binding is present. Second, JONES binding is absent in the EGL of the intermediate folia of the P0 cerebellum, where cell division is pronounced but the inward migration of the granule cells has not yet occurred. Finally, earlier observations in embryonic retina showed JONES immunoreactivity to be absent from the purely proliferative ciliary margin until the relatively latter embryonic stages, when that region begins to generate the first retinal neurons (Constantine-Paton et al., 1986).

In this paper we have also compared the distribution of the antigens recognized by the JONES antibody with the staining pattern of the anti-D.1.1 antibody. We have shown that, in forebrain and in cerebellum, both antibodies had a similar pattern of staining and that this labeling was not restricted to the proliferative zone; rather, it is better correlated with the ventricular zone and intermediate region where postmitotic cells are migrating (see Fig. 12). To explain the differences between our results and those published earlier in relation to the pattern of staining with the D.1.1 antibody, we suggest that treatment of the tissue with Triton X-100 probably affects the immunoreactivity such that the antigen can be visualized only in the heavily stained regions such as the ventricular zone.

\section{Localization of JONES binding in the adult brain}

JONES immunocytochemical reactivity is absent in all regions of the adult brain except the retina and the cerebellum. In addition, the pattern of JONES immunorcactivity, where present in the adult, is qualitatively as well as quantitatively different from the patterns of reactivity observed in the developing animal. Thus, in the adult, JONES binds to synapse-rich regions (e.g., the molecular layer of the cerebellum and the outer plexiform layer of the retina) that are not present in the developing animal. However, it also binds to regions, such as the optic nerve head, that do not show JONES immunoreactivity during development. These observations suggest that the function of the JONES antigens may, in fact, he quite different in adult and developing nervous tissue. The idea is supported by our consistent localization of JONES binding to kidney glomeruli, where any association with neural tissue is unlikely.

The major conclusions of this study can be summarized as follows. First, the functions served by the JONES antigens in the adult brain and kidney are likely to be different from the functions served in the developing animal. Second, though JONES binding overlaps extensively with regions of the developing brain where neuronal or glial proliferation is occurring, it does not appear to be associated with cell division per se. Third, in all regions studied JONES binding colocalizes extremely well with populations of migrating cells or with regions where axon pathways are in the process of being established. The fact that JONES immunorcactivity is not present in all regions of the developing nervous system where migration is occurring indicates that the molecules recognized by the JONES mab are probably not crucial components of the cellular machinery involved in cell motility. This observation suggests that the JONES antigens may play an instructive, rather than a permissive role, in orchestrating the patterns of cell an axon migration that bring about the topography and cytoarchitecture of the mature brain.

\section{References}

Altman, J. (1966) Autoradiographic and histological studies of postnatal neurogenesis. II. A longitudinal investigation of the kinetics, migration and transformation of cells incorporating tritiated thymidine in infant rats, with special reference to postnatal neurogenesis in some brain regions. J. Comp. Neurol. 128: 431-473.

Altman, J. (1969) Autoradiographic and histological studies of postnatal neurogenesis. III. Dating the time of production and onset of differentiation of cerebellar microneurons in rats. J. Comp. Neurol. 136: 269-294.

Altman, J., and S. A. Bayer (1984) The development of the rat spinal cord. Adv. Anat. Embryol. Cell Biol. 85: 1-166.

Altman, J., and S. A. Bayer (1985a) Embryonic development of the rat cerebellum. I. Delineation of the cerebellar primordium and early cell movements. J. Comp. Neurol. 231: 1-26.

Altman, J., and S. A. Bayer (1985b) Embryonic development of the rat cerebellum. II. Translocation and regional distribution of the deep neurons. J. Comp. Neurol. 231: 27-41.

Altman, J., and S. A. Bayer (1985c) Embryonic development of the rat cerebellum. III. Regional differences in the time of origin, migration, and settling of Purkinje cells. J. Comp. Neurol 231: 42-65.

Altman, J., and G. D. Das (1965) Autoradiographic and histological evidence of postnatal hippocampal neurogenesis in rats. J. Comp. Neurol. 124: 319-336.

Beasley, L., and W. B. Stallcup (1987) The nerve growth factor-inducible large external (NILE) glycoprotein and neural cell adhesion molecule (N-CAM) have distinct patterns of expression in the developing rat central nervous system. J. Neurosci. 7: 708-715.

Blackburn, C. C., P. Swank-Hill, and R. L. Schnaar (1986) Gangliosides support neural retina cell adhesion. J. Biol. Chem. 261: 28732881.

Blum, A. S., and C. J. Barnstable (1986) Structure and regulation of a developmentally restricted neuronal surface antigen. J. Cell Biol. 103: 231a.

Braekevelt, C. R., and M. J. Hollenberg (1970) The development of the retina of the albino rat. Am. J. Anat. 127: 281-302.

Bruckner, G., V. Mares, and D. Biesold (1976) Neurogenesis in the visual system of the rat. An autoradiographic investigation. J. Comp. Neurol. 166: 245-256.

Bunt, S. M., R. D. Lund, and P. W. Land (1983) Prenatal development of the optic projection in albino and hood rats. Dev. Brain Res. 6 . 149-168.

Cheresh, d. A., A. P. Varki, N. M. Varki, W. B. Stallcup, J. Levine, and R. A. Reisfeld (1984) A monoclonal antibody recognizes an 
$\mathrm{O}$-acetylated sialic acid in a human melanoma-associated ganglioside J. Biol. Chem. 259: 7453-7459.

Constantine-Paton, M., A. S. Blum, R. Mendez-Otero, and C. J. Barnstable (1986) A cell surface molecule distributed in a dorsoventral gradient in the perinatal rat retina. Nature, 324: 459-462.

Goldman, J. E., M. Hirano, R. K. Yu, and T. N. Seyfried (1984) GD3 ganglioside is a glycolipid characteristic of immature neuroectodermal cells. J. Neuroimmunol. 7: 179-192.

Gottlieb, D. I., and L. Glaser (1980) Cellular recognition during neural development. Annu. Rev. Neurosci. 3: 303-318.

Hakomori, S. (1981) Glycosphingolipids in cellular interaction, differentiation and oncogenesis. Annu. Rev. Biochem. 50: 733-764.

Hicks, S. P., and C. J. D'Amato (1968) Cell migrations to the isocortex in the rat. Anat. Rec. 160: 619-634.

Hockfield, S., and R. D. G. McKay (1985) Identification of major cell classes in the developing mammalian nervous system. J. Neurosci. 5: 3310-3328.

Horsburgh, G. M., and A. J. Sefton (1986) The early development of the optic nerve and chiasm in the embryonic rat. J. Comp. Neurol. 243: $547-560$.

Kuwabara, T., and T. A. Weidman (1974) Development of the prenatal rat retina. Invest. Ophtalmol. 13: 725-739.

Laemmli, U. (1970) Cleavage of structural proteins during the assembly of the bacteriophage T4. Nature 227: 680-685.

Levine, J. M., L. Beasley, and W. B. Stallcup (1984) The D1.1 antigen; a cell surface marker for germinal cells of the central nervous system. J. Neurosci. 4: 820-831.

Levine, J. M., L. Beasley, and W. B. Stallcup (1986) Localization of a neuroectoderm-associated cell surface antigen in the developing and adult rat. Dev. Brain Res. 27: 211-222.

Lund, R., and A. H. Bunt (1976) Prenatal development of central optic pathways in albino rats. J. Comp. Neurol. 165: 247-264.

Lund, R., and M. J. Mustari (1977) Development of the geniculocortical pathway in rats. J. Comp. Neurol. 173: 289-306.

Marchase, R. B. (1977) Biochemical investigation of retinotectal adhesive specificity. J. Cell Biol. 75: 237-257.
Maslim, J., M. Webster, and J. Stone (1986) Stages in the structural differentiation of retinal ganglion cells. J. Comp. Neurol. 254: 382402.

Mendez-Otero, R, B. Schlosshauer, and M. Constantine-Paton (1986) JONES binding gangliosides are associated with regions of cell and process migration in the developing CNS. Soc. Neurosci. Abstr. 12: 317.

Ouchterlony, O., and C. A. Nilsson (1978) Immunodiffusion immunoelectrophoresis. In Handbook of Experimental Immunology, D. M. Weir, ed., pp. 1-44, Blackwell Scientific, Oxford, U.K.

Raedler, A., and J. Sicvers (1975) The development of the visual system of the albino rat. Adv. Anat. Embryol. Cell Biol. 50: 1-87.

Raedler, A., and J. Sievers (1976) Light and electron microscopical studies on specific cells of the marginal zone in the developing rat cerebral cortex. Anat. Embryol. 149: 173-181.

Rakic, P. (1971) Neuron-glia relationship during granule cell migration in developing cerebellar cortex. A Golgi and electronmicroscopic study in Macacus Rhesus. J. Comp. Neurol. 141: 283-312.

Schlosshauer, B., R. Mendez-Otero, and M. Constantine-Paton (1986) Developmental regulation of JONES gangliosides in the mammalian nervous system. Soc. Neurosci. Abstr. 12: 317.

Schlosshauer, B., A. S. Blum, R. Mendez-Otero, C. J. Barnstable, and M. Constantine-Paton (1988) Developmental regulation of ganglioside antigens recognized by the JONES antibody. J. Neurosci. 8: 580592.

Silver, J., and J. Sapiro (1981) Axonal guidance during development of the optic nerve: The role of pigmented epithelia and other extrinsic factors. J. Comp. Neurol. 202: 521-538.

Stallcup, W. B., L. Beasly, and J. Levine (1983) Cell surface molecules that characterize different stages in the development of cerebellar interneurons. Cold Spring Harbor Symp. Quant. Biol. 48: 761-774.

Valentino, K. L., J. Winter, and L. F. Reichart (1985) Applications of monoclonal antibodies to neuroscience research. Annu. Rev. Neurosci. 8: 199-232.

Weidman, T. A., and T. Kuwabara (1968) Postnatal development of the rat retina. Arch. Ophthalmol. 79: 470-484. 